\title{
A LOCAL MONOTONICITY FORMULA FOR AN INHOMOGENOUS SINGULAR PERTURBATION PROBLEM AND APPLICATIONS
}

\author{
CLAUDIA LEDERMAN AND NOEMI WOLANSKI
}

\begin{abstract}
In this paper we prove a local monotonicity formula for solutions to an inhomogeneous singularly perturbed diffusion problem of interest in combustion. This type of monotonicity formula has proved to be very useful for the study of the regularity of limits $u$ of solutions of the singular perturbation problem and of $\partial\{u>0\}$, in the global homogeneous case.

As a consequence of this formula we prove that $u$ has an asymptotic development at every point in $\partial\{u>0\}$ where there is a nonhorizontal tangent ball. These type of developments have been essential for the proof of the regularity of $\partial\{u>0\}$ for Bernoulli and Stefan free boundary problems.

We also present applications of our results to the study of $\partial\{u>0\}$ in the stationary case including, in particular, its regularity in the case of energy minimizers. We present as well a regularity result for travelling waves of a combustion model that relies on our monotonicity formula and its consequences.

The fact that our results hold for the inhomogeneous problem allows a very wide applicability. In fact, they may be applied to problems with nonlocal diffusion and/or transport.
\end{abstract}

\section{INTRODUCTION}

In this paper we develop a local monotonicity formula for solutions to an inhomogeneous singular perturbation problem of interest in combustion theory. It is based on a global monotonicity formula that G. S. Weiss developed for solutions of the global homogeneous problem (see [18]). The problem under consideration here is the following: for $\varepsilon>0$ we let $u^{\varepsilon}$ be a family of solutions to

$$
\left(P_{\varepsilon}\left(f_{\varepsilon}\right)\right) \quad \Delta u^{\varepsilon}-u_{t}^{\varepsilon}=\beta_{\varepsilon}\left(u^{\varepsilon}\right)+f_{\varepsilon} \text { in } \mathcal{D}
$$

where $\varepsilon>0, \mathcal{D}$ is a domain in $\mathbb{R}^{N+1}, f_{\varepsilon}$ are uniformly bounded in $L^{\infty}(\mathcal{D}), \beta_{\varepsilon}(s)=\frac{1}{\varepsilon} \beta\left(\frac{s}{\varepsilon}\right)$ with $\beta$ a Lipschitz continuous function, $\beta(s)>0$ for $0<s<1$ and $\beta(s)=0$ otherwise. This type of reaction term appears in the study of the propagation of deflagration flames. In this context $\varepsilon$ represents the inverse of the activation energy (see for instance, [2], [3], [15] and the references therein).

We prove that $u^{\varepsilon}$ satisfies a local monotonicity formula. This formula allows us to state our problem in bounded domains and not only in $\mathbb{R}^{N} \times(0, T)$ as considered in [18]. Moreover, we show that the formula holds for the inhomogeneous equation, this is, $f_{\varepsilon} \not \equiv 0$.

As an application of this formula, we prove that any limit $u$ of solutions $u^{\varepsilon}$ of this singular perturbation problem (as $\varepsilon \rightarrow 0$ ) has an asymptotic development at each point on $\partial\{u>0\}$

1991 Mathematics Subject Classification. 35R35, 35J65, 80A25, 35B65.

Key words and phrases. Singular perturbation problems, monotonicity formula, inhomogeneous problems, combustion.

The research of the authors was partially supported by Fundación Antorchas Project 13900-5, Universidad de Buenos Aires grant X052, ANPCyT PICT No 03-13719, CONICET PIP 5478. The authors are members of CONICET . 
where there is a nonhorizontal tangent ball contained either in $\{u>0\}$ or in $\{u \leq 0\}^{\circ}$, and we determine all the slopes that it is possible to encounter in this way. Moreover, we present applications of the results in this paper to the study of the regularity of the boundary of $\{u>0\}$ (the flame front in combustion models).

The fact that we are looking at the inhomogeneous equation makes the applicability of these results much wider. In particular, the results in this paper apply to more general equations that include nonlocal diffusion and/or transport. In fact, let $u^{\varepsilon}=u^{\varepsilon}(x, t)$ be a family of solutions to:

$$
\begin{aligned}
& (1-\theta) \int J(x-y)\left(u^{\varepsilon}(y, t)-u^{\varepsilon}(x, t)\right) d y+\theta \Delta u^{\varepsilon}+ \\
& \quad+V_{\varepsilon}(x, t) \cdot \nabla u^{\varepsilon}+c_{\varepsilon}(x, t) u^{\varepsilon}-u_{t}^{\varepsilon}=\beta_{\varepsilon}\left(u^{\varepsilon}\right)+g_{\varepsilon}
\end{aligned}
$$

where $\beta_{\varepsilon}$ is as above, $0<\theta \leq 1, J \in L^{1}\left(\mathbb{R}^{N}\right)$ with $J(x)=J(-x), J \geq 0$, and with $V_{\varepsilon} \in\left(L^{\infty}\right)^{N}$, $c_{\varepsilon} \in L^{\infty}, g_{\varepsilon} \in L^{\infty}$ all with norms uniformly bounded with respect to $\varepsilon$ (the term involving the integral in $G_{\varepsilon}\left(g_{\varepsilon}\right)$ is used to model nonlocal diffusion, see [12]). Then, $u^{\varepsilon}$ satisfy

$$
\theta \Delta u^{\varepsilon}-u_{t}^{\varepsilon}=\beta_{\varepsilon}\left(u^{\varepsilon}\right)+f_{\varepsilon}
$$

with

$$
f_{\varepsilon}=g_{\varepsilon}-V_{\varepsilon}(x, t) \cdot \nabla u^{\varepsilon}-c_{\varepsilon}(x, t) u^{\varepsilon}-(1-\theta) \int J(x-y)\left(u^{\varepsilon}(y, t)-u^{\varepsilon}(x, t)\right) d y .
$$

In this paper we prove the monotonicity formula for a family of solutions to $P_{\varepsilon}\left(f_{\varepsilon}\right)$ under the assumption that the solutions $u^{\varepsilon}$ are uniformly bounded in $\operatorname{Lip}(1,1 / 2)$ norm and that $\left\|f_{\varepsilon}\right\|_{L^{\infty}}$ are uniformly bounded.

In case $u^{\varepsilon}$ are solutions to $G_{\varepsilon}\left(g_{\varepsilon}\right)$ which are uniformly bounded in Lip $(1,1 / 2)$ norm, the functions $f_{\varepsilon}$ given by (1.1) are uniformly bounded in $L^{\infty}$ norm. So $u^{\varepsilon}$ are in the situation considered in this paper.

Local bounds for the gradients of solutions $u^{\varepsilon}$ to $G_{\varepsilon}\left(g_{\varepsilon}\right)$ in terms of bounds of $\left\|u^{\varepsilon}\right\|_{L^{\infty}}$ were proved in different contexts. For instance, for $V_{\varepsilon}=0$ they were proved in [13] in the one phase case (i.e. $u^{\varepsilon} \geq 0$ ) and in [14] in the stationary two phase case. For $\theta=1$ they were proved in the stationary one phase case in [2], when $g_{\varepsilon}=0$; in the evolutionary two phase case in [6], when $V_{\varepsilon}=0, c_{\varepsilon}=0$ and $g_{\varepsilon}=0$; and in [8], when $g_{\varepsilon}=0$. Moreover, in global situations for initial data uniformly bounded in Lipschitz norm, it is possible to obtain these bounds by applying Bernstein type techniques, as done in [11] and [18] when $\theta=1, V_{\varepsilon}=0, c_{\varepsilon}=0, g_{\varepsilon}=0$ and $u^{\varepsilon} \geq 0$. Once the bound on the gradients is known, the bound on the Hölder $1 / 2$ seminorm in the $t$ variable is easily obtained (see, for instance, [9] for the proof).

The main results in the paper are two local monotonicity formulae: one for solutions $u^{\varepsilon}$ of problem $P_{\varepsilon}\left(f_{\varepsilon}\right)$ (Theorem 2.1) and one for the limits (as $\left.\varepsilon \rightarrow 0\right) u$ of $u^{\varepsilon}$ (Theorem 2.2).

The second one, in particular, says that if $u=\lim u^{\varepsilon}, \chi=\lim B_{\varepsilon}\left(u^{\varepsilon}\right),\left(x_{0}, t_{0}\right) \in \partial\{u>0\}$ and $\psi=\psi(x) \in C_{0}^{\infty}, 0 \leq \psi \leq 1, \psi \equiv 1$ in a neighborhood of $x_{0}$, then there exists

$$
\delta\left(x_{0}, t_{0}\right)=\lim _{r \rightarrow 0} \frac{1}{r^{2}} \int_{t_{0}-4 r^{2}}^{t_{0}-r^{2}} \int_{\mathbb{R}^{N}}\left(|\nabla(u \psi)|^{2}+2 \psi^{2} \chi+\frac{1}{2} \frac{(u \psi)^{2}}{t-t_{0}}\right) G\left(x-x_{0}, t_{0}-t\right) d x d t,
$$

where $G(x, t)=\frac{1}{(4 \pi t)^{N / 2}} \exp \left(-\frac{|x|^{2}}{4 t}\right)$.

As a corollary to these results we obtain that any blow up limit $u_{0}$ of $u=\lim u^{\varepsilon}$ at a point $\left(x_{0}, t_{0}\right) \in \partial\{u>0\}\left(u_{0}=\lim _{\lambda_{n} \rightarrow 0} \frac{1}{\lambda_{n}} u\left(x_{0}+\lambda_{n} x, t_{0}+\lambda_{n}^{2} t\right)\right)$ is homogeneous for $t \leq 0$. This is, $u_{0}\left(r x, r^{2} t\right)=r u_{0}(x, t)$, for $t \leq 0$ and any $r>0$ (Corollary 2.1). 
The knowledge of homogeneity properties of blow up limits is a main step in the classification of those limits, which is a key tool towards the understanding of the behavior of $u$ near $\partial\{u>0\}$ in free boundary problems. See, for instance, $[10,17]$ for the obstacle problem, $[7,16]$ for Bernoulli's problem, and Sections 3 and 4 below for the problem under consideration in the present paper.

A second corollary is that, given $u=\lim u^{\varepsilon}, \chi=\lim B_{\varepsilon}\left(u^{\varepsilon}\right),\left(x_{0}, t_{0}\right) \in \partial\{u>0\}$ and $\delta\left(x_{0}, t_{0}\right)$ as in (1.2) then, for any sequence $\lambda_{n} \rightarrow 0$, and limits $u_{0}=\lim \frac{1}{\lambda_{n}} u\left(x_{0}+\lambda_{n} x, t_{0}+\lambda_{n}^{2} t\right)$ and $\chi_{0}=\lim \chi\left(x_{0}+\lambda_{n} x, t_{0}+\lambda_{n}^{2} t\right)$, there holds that, for any $r>0$,

$$
\begin{aligned}
\delta\left(x_{0}, t_{0}\right) & =\frac{1}{r^{2}} \int_{-4 r^{2}}^{-r^{2}} \int_{\mathbb{R}^{N}}\left(\left|\nabla u_{0}\right|^{2}+2 \chi_{0}+\frac{1}{2} \frac{u_{0}^{2}}{t}\right) G(x,-t) d x d t \\
& =\frac{1}{r^{2}} \int_{-4 r^{2}}^{-r^{2}} \int_{\mathbb{R}^{N}} 2 \chi_{0} G(x,-t) d x d t
\end{aligned}
$$

and $0 \leq \delta\left(x_{0}, t_{0}\right) \leq 6 M$ where $M=\int \beta$ (Corollary 2.2$)$.

From (1.3) (see Remark 2.3) it is possible to see that the value of $\delta\left(x_{0}, t_{0}\right)$ is intimately related to the regularity of $\partial\{u>0\}$ in a neighborhood of $\left(x_{0}, t_{0}\right)$. Moreover, we prove in Theorem 9.7 in [14], which holds in the stationary case, that $\delta\left(x_{0}\right)=3 M$ if and only if $\partial\{u>0\}$ is smooth in a neighborhood of $x_{0}$ (see also Section 4 in this paper).

Concerning the applications of our monotonicity formula and its corollaries, we present in this paper two type results.

The result on asymptotic developments described above (Theorem 3.1 ) concerns limits $u$ of solutions $u^{\varepsilon}$ to the singular perturbation problem $P_{\varepsilon}\left(f_{\varepsilon}\right)$. These limits are solutions to a differential equation in $\{u>0\}$ and $\{u \leq 0\}^{\circ}$ (see for instance, [14]). Thus, a thorough understanding of the limits $u$ comes from the understanding of the boundaries $\partial\{u>0\}$. The asymptotic developments we obtain in Theorem 3.1 are a step towards this end. In fact, asymptotic developments like the ones in this paper have proved to be very useful to obtain regularity results of $\partial\{u>0\}$ for free boundary problems (see for instance, $[1,4,5]$ ). In these papers the knowledge of the asymptotic behavior of $u$ at points of $\partial\{u>0\}$ with a nonhorizontal tangent ball has proved to be essential.

Moreover, in this paper we characterize all the possible slopes at which $u$ may leave $\partial\{u>0\}$. All these cases can happen as was shown with examples in [13]. So that our results are really sharp.

One of the main tools in the proof of Theorem 3.1 is a result on the asymptotic development at nonhorizontal points $\left(x_{0}, t_{0}\right)$ in the boundary of a ball $B$ at which $u\left(x_{0}, t_{0}\right)=0$, for nonnegative functions satisfying either $\Delta u-u_{t} \leq C$ in $B$ with $u>0$ in $B$, or $\Delta u-u_{t} \geq-C$ in $B^{c}$ with $u \equiv 0$ in $B$. These asymptotic developments were proved by the authors in a previous paper (see [13]).

We point out that the result in Theorem 3.1 is new even for the global problem and/or with $f_{\varepsilon} \equiv 0$.

The second type of application we present here deals with the regularity of the boundary of $\{u>0\}$ in the stationary case including, in particular, regularity results for energy minimizers and also with regularity results for travelling waves of a combustion model. 
In particular, in many situations we conclude that if $u^{\varepsilon_{j}}$ are stationary solutions to $P_{\varepsilon_{j}}\left(f_{\varepsilon_{j}}\right)$ and $\varepsilon_{j} \rightarrow 0$ then, $u=\lim u^{\varepsilon_{j}}$ is a classical solution to the following free boundary problem

$$
\begin{array}{ll}
\Delta u=f \chi_{\{u \neq 0\}} & \text { in }\{u>0\} \cup\{u \leq 0\}^{\circ}, \\
\left|\nabla u^{+}\right|^{2}-\left|\nabla u^{-}\right|^{2}=2 M & \text { on } \partial\{u>0\} .
\end{array}
$$

Concerning the monotonicity formula proved in this paper, it builds up from a formula introduced by G. S. Weiss in [18] for the global homogeneous version of the problem considered here. The formula was a crucial tool in [18] to obtain rectificability results for $\partial\{u>0\}$.

The paper is organized as follows. In Section 2 we prove the local monotonicity formulae for the singular perturbation problem $P_{\varepsilon}\left(f_{\varepsilon}\right)$ and its limits. Then, we derive some consequences of them. In particular, we prove some corollaries that imply that for blow up limits $u_{0}$ of limit functions $u$, the global functional of Weiss (cf. [18]) is constant and $u_{0}$ is homogeneous. In Section 3 we use these results to obtain asymptotic developments at points on $\partial\{u>0\}$. In Section 4 we present applications to regularity results of $\partial\{u>0\}$ that are proved by the authors in [14] using the results in this paper.

Notation. Throughout the paper $\mathcal{D}$ will be a domain in $\mathbb{R}^{N+1}$. We will be considering rescalings of functions and we will denote

$$
v_{r}(x, t)=\frac{1}{r} v\left(r x, r^{2} t\right) \quad \text { and } \quad v^{r}(x, t)=v\left(r x, r^{2} t\right) .
$$

We will say that a function $v$ is in the class $\operatorname{Lip}(1,1 / 2)$ in a domain $\mathcal{D}$, if $v$ is bounded and there exists a constant $L=L(\mathcal{D})$ such that

$$
|v(x, t)-v(y, \tau)| \leq L\left(|x-y|+|t-\tau|^{\frac{1}{2}}\right)
$$

for every $(x, t),(y, \tau)$ in $\mathcal{D}$. The norm in $\operatorname{Lip}(1,1 / 2)$ in $\mathcal{D}$ is

$$
\|v\|_{L i p(1,1 / 2)}=\|v\|_{L^{\infty}(\mathcal{D})}+\sup _{(x, t),(y, \tau) \in \mathcal{D}} \frac{|v(x, t)-v(y, \tau)|}{|x-y|+|t-\tau|^{1 / 2}} .
$$

We denote by

$$
|v|_{\operatorname{Lip}(1,1 / 2)}=\sup _{(x, t),(y, \tau) \in \mathcal{D}} \frac{|v(x, t)-v(y, \tau)|}{|x-y|+|t-\tau|^{1 / 2}}
$$

the $\operatorname{Lip}(1,1 / 2)$ seminorm in $\mathcal{D}$.

Finally, we will denote $B_{\varepsilon}(r)=\int_{0}^{r} \beta_{\varepsilon}(s) d s, M=\int_{0}^{1} \beta(s) d s$ and $G(x, t)=\frac{1}{(4 \pi t)^{N / 2}} \exp \left(-\frac{|x|^{2}}{4 t}\right)$.

\section{Monotonicity Formula}

In this section we prove the local monotonicity formulae for the singular perturbation problem $P_{\varepsilon}\left(f_{\varepsilon}\right)$ and its limits (Theorems 2.1 and 2.2). Then we derive some consequences of them.

We first obtain

Theorem 2.1 ( $\varepsilon$-Monotonicity Formula). Let $u^{\varepsilon}$ be a family of solutions to $P_{\varepsilon}\left(f_{\varepsilon}\right)$ in $\mathcal{D}$, uniformly bounded in Lip $(1,1 / 2)$ with $f_{\varepsilon}$ uniformly bounded in $L^{\infty}$ norm in $\mathcal{D}$ and $B_{R}(0) \times$ $(-4,0) \subset \subset \mathcal{D}$. For $\psi=\psi(x) \in C_{0}^{\infty}\left(B_{R}(0)\right), 0 \leq \psi \leq 1, \psi \equiv 1$ in $B_{R / 2}(0)$ and $0<r \leq 1$, let 


$$
\begin{aligned}
& \mathcal{W}^{\varepsilon}(r)=\mathcal{W}^{\varepsilon}\left(r, u^{\varepsilon}, \psi\right)= \\
& \quad=\frac{1}{r^{2}} \int_{-4 r^{2}}^{-r^{2}} \int_{\mathbb{R}^{N}}\left(\left|\nabla\left(u^{\varepsilon} \psi\right)\right|^{2}+2 \psi^{2} B_{\varepsilon}\left(u^{\varepsilon}\right)+\frac{1}{2} \frac{\left(u^{\varepsilon} \psi\right)^{2}}{t}\right) G(x,-t) d x d t
\end{aligned}
$$

where $G(x, t)=\frac{1}{(4 \pi t)^{N / 2}} \exp \left(-\frac{|x|^{2}}{4 t}\right)$.

Then,

$$
\begin{aligned}
\frac{\partial \mathcal{W}^{\varepsilon}}{\partial r}(r) \geq & \int_{-4}^{-1} \int_{\mathbb{R}^{N}}\left(\partial_{r} w_{r}^{\varepsilon}\right)^{2} \frac{r G(x,-t)}{-t} d x d t- \\
& -C_{1}\left(\left\|f_{\varepsilon}\right\|_{L^{\infty}\left(B_{R}(0) \times(-4,0)\right)}+\left\|f_{\varepsilon}\right\|_{L^{\infty}\left(B_{R}(0) \times(-4,0)\right)}^{3 / 2}\right)- \\
& -C_{2}\left\|f_{\varepsilon}\right\|_{L^{\infty}\left(B_{R}(0) \times(-4,0)\right)} \frac{\left|u^{\varepsilon}(0,0)\right|}{r}-C_{3} C(R) e^{-C_{4} \frac{R^{2}}{r^{2}}} .
\end{aligned}
$$

Here $w^{\varepsilon}(x, t)=\psi(x) u^{\varepsilon}(x, t)$ and $C(R)=\max \left\{\frac{1}{R}, \frac{1}{R^{N / 2}}\right\}$.

Let $A_{i}, i=1, \cdots, 4$ be such that

$$
\begin{array}{lll}
\left\|u^{\varepsilon}\right\|_{L^{\infty}\left(B_{R}(0) \times(-4,0)\right)} \leq A_{1} & , \quad\left|u^{\varepsilon}\right|_{L_{i p_{(1,1 / 2)}\left(B_{R}(0) \times(-4,0)\right)} \leq A_{2}} \\
\left\|u_{t}^{\varepsilon}\right\|_{L^{2}\left(B_{R}(0) \times(-4,0)\right)} \leq A_{3} & , \quad & \|\psi\|_{C^{2}\left(B_{R}(0)\right)} \leq A_{4}
\end{array}
$$

The constants $C_{i}$ in (2.2) depend only on $A_{i}$, the dimension $N, M_{1}=\max _{0 \leq s \leq 1} s \beta(s)$ and $M=\int_{0}^{1} \beta(s) d s$.

Remark 2.1. Observe that in [13] it was proved in the one phase case that solutions $u^{\varepsilon}$ to $P_{\varepsilon}\left(f_{\varepsilon}\right)$ with uniformly bounded Lip $(1,1 / 2)$ norm and $\left\|f_{\varepsilon}\right\|_{L^{\infty}}$ uniformly bounded, in a domain $\mathcal{D}^{\prime} \supset \supset \mathcal{D}$, have uniformly bounded $\left\|u_{t}^{\varepsilon}\right\|_{L^{2}(\mathcal{D})}$ and satisfy that subsequences of $\nabla u^{\varepsilon}$ converge strongly in $L^{2}\left(\mathcal{D}^{\prime}\right)$. The same proof holds for the two phase case.

Proof of the theorem. By rescaling we get

$$
\begin{aligned}
\mathcal{W}^{\varepsilon}(r) & = \\
& =\int_{-4}^{-1} \int_{\mathbb{R}^{N}}\left(\left|\nabla w_{r}^{\varepsilon}\right|^{2}+2\left(\psi^{r}\right)^{2} B_{\varepsilon}\left(r u_{r}^{\varepsilon}\right)+\frac{1}{2} \frac{\left(w_{r}^{\varepsilon}\right)^{2}}{t}\right) G(x,-t) d x d t .
\end{aligned}
$$

Then,

$$
\begin{aligned}
\frac{\partial \mathcal{W}^{\varepsilon}}{\partial r}(r)=\int_{-4}^{-1} \int_{\mathbb{R}^{N}}\left(2 \nabla w_{r}^{\varepsilon} \nabla\left(\partial_{r} w_{r}^{\varepsilon}\right)+2\left(\psi^{r}\right)^{2} \beta_{\varepsilon}\left(r u_{r}^{\varepsilon}\right)\left(u_{r}^{\varepsilon}+r \partial_{r} u_{r}^{\varepsilon}\right)+\right. \\
\left.\quad+4 \psi^{r} \partial_{r} \psi^{r} B_{\varepsilon}\left(r u_{r}^{\varepsilon}\right)+\frac{1}{t} w_{r}^{\varepsilon} \partial_{r} w_{r}^{\varepsilon}\right) G(x,-t) d x d t \geq \\
\geq \int_{-4}^{-1} \int_{\mathbb{R}^{N}}\left(-2 \Delta w_{r}^{\varepsilon} \partial_{r} w_{r}^{\varepsilon}+2\left(\psi^{r}\right)^{2} \beta_{\varepsilon}\left(r u_{r}^{\varepsilon}\right) r \partial_{r} u_{r}^{\varepsilon}+\right. \\
\left.\quad+4 \psi^{r} \partial_{r} \psi^{r} B_{\varepsilon}\left(r u_{r}^{\varepsilon}\right)+\frac{1}{t} w_{r}^{\varepsilon} \partial_{r} w_{r}^{\varepsilon}-2 \partial_{r} w_{r}^{\varepsilon} \frac{x}{2 t} \cdot \nabla w_{r}^{\varepsilon}\right) G(x,-t) d x d t
\end{aligned}
$$

since $u_{r}^{\varepsilon} \beta_{\varepsilon}\left(r u_{r}^{\varepsilon}\right) \geq 0$ and $\nabla G(x,-t)=\frac{x}{2 t} G(x,-t)$. Thus, 


$$
\begin{aligned}
& \frac{\partial \mathcal{W}^{\varepsilon}}{\partial r}(r) \geq \int_{-4}^{-1} \int_{\mathbb{R}^{N}}\left(\partial_{r} w_{r}^{\varepsilon}\left(-2 \Delta w_{r}^{\varepsilon}+\frac{1}{t} w_{r}^{\varepsilon}-2 \frac{x}{2 t} \cdot \nabla w_{r}^{\varepsilon}\right)+2\left(\psi^{r}\right)^{2} \beta_{\varepsilon}\left(r u_{r}^{\varepsilon}\right) r \partial_{r} u_{r}^{\varepsilon}+\right. \\
& \left.+4 \psi^{r} \partial_{r} \psi^{r} B_{\varepsilon}\left(r u_{r}^{\varepsilon}\right)\right) G(x,-t) d x d t \\
& =\int_{-4}^{-1} \int_{\mathbb{R}^{N}}\left(\partial_{r} w_{r}^{\varepsilon}\left(-2 \psi^{r}\left(\Delta-\partial_{t}\right) u_{r}^{\varepsilon}-2 u_{r}^{\varepsilon} \Delta \psi^{r}-4 \nabla \psi^{r} \nabla u_{r}^{\varepsilon}-\frac{r}{t} \partial_{r} w_{r}^{\varepsilon}\right)\right. \\
& \left.+2 \psi^{r} r \beta_{\varepsilon}\left(r u_{r}^{\varepsilon}\right)\left(\partial_{r} w_{r}^{\varepsilon}-u_{r}^{\varepsilon} \partial_{r} \psi^{r}\right)+4 \psi^{r} \partial_{r} \psi^{r} B_{\varepsilon}\left(r u_{r}^{\varepsilon}\right)\right) G(x,-t) d x d t \\
& =\int_{-4}^{-1} \int_{\mathbb{R}^{N}}\left(\partial_{r} w_{r}^{\varepsilon}\left(-2 \psi^{r} r f_{\varepsilon}^{r}-2 u_{r}^{\varepsilon} \Delta \psi^{r}-4 \nabla \psi^{r} \nabla u_{r}^{\varepsilon}-\frac{r}{t} \partial_{r} w_{r}^{\varepsilon}\right)\right. \\
& \left.-2 \psi^{r} \beta_{\varepsilon}\left(r u_{r}^{\varepsilon}\right) r u_{r}^{\varepsilon} \partial_{r} \psi^{r}+4 \psi^{r} \partial_{r} \psi^{r} B_{\varepsilon}\left(r u_{r}^{\varepsilon}\right)\right) G(x,-t) d x d t .
\end{aligned}
$$

We have used that

$$
\begin{gathered}
-2 \Delta w_{r}^{\varepsilon}-2 \frac{x}{2 t} \cdot \nabla w_{r}^{\varepsilon}+\frac{w_{r}^{\varepsilon}}{t}=-2\left(\Delta-\partial_{t}\right) w_{r}^{\varepsilon}-2 \partial_{t} w_{r}^{\varepsilon}-\frac{x}{t} \cdot \nabla w_{r}^{\varepsilon}+\frac{w_{r}^{\varepsilon}}{t}= \\
=-2\left(\Delta-\partial_{t}\right) w_{r}^{\varepsilon}-\frac{r}{t} \partial_{r} w_{r}^{\varepsilon}=-2 \psi^{r}\left(\Delta-\partial_{t}\right) u_{r}^{\varepsilon}-2 u_{r}^{\varepsilon} \Delta \psi^{r}-4 \nabla \psi^{r} \nabla u_{r}^{\varepsilon}-\frac{r}{t} \partial_{r} w_{r}^{\varepsilon}, \\
\psi^{r} \partial_{r} u_{r}^{\varepsilon}=\partial_{r} w_{r}^{\varepsilon}-u_{r}^{\varepsilon} \partial_{r} \psi^{r}, \quad \text { and } \quad\left(\Delta-\partial_{t}\right) u_{r}^{\varepsilon}=r \beta_{\varepsilon}\left(r u_{r}^{\varepsilon}\right)+r f_{\varepsilon}^{r} .
\end{gathered}
$$

Therefore,

$$
\begin{aligned}
\frac{\partial \mathcal{W}^{\varepsilon}}{\partial r}(r) & \geq \int_{-4}^{-1} \int_{\mathbb{R}^{N}}\left(\partial_{r} w_{r}^{\varepsilon}\right)^{2} \frac{r G(x,-t)}{-t} d x d t+ \\
& +\int_{-4}^{-1} \int_{\mathbb{R}^{N}}\left(\partial_{r} w_{r}^{\varepsilon}\right)\left(-2 \psi^{r} r f_{\varepsilon}^{r}\right) G(x,-t) d x d t+ \\
& +\int_{-4}^{-1} \int_{\mathbb{R}^{N}}\left(\partial_{r} w_{r}^{\varepsilon}\right)\left(-2 u_{r}^{\varepsilon} \Delta \psi^{r}-4 \nabla \psi^{r} \nabla u_{r}^{\varepsilon}\right) G(x,-t) d x d t+ \\
& +\int_{-4}^{-1} \int_{\mathbb{R}^{N}}\left(-2 \psi^{r} \beta_{\varepsilon}\left(r u_{r}^{\varepsilon}\right) r u_{r}^{\varepsilon} \partial_{r} \psi^{r}+4 \psi^{r} \partial_{r} \psi^{r} B_{\varepsilon}\left(r u_{r}^{\varepsilon}\right)\right) G(x,-t) d x d t= \\
& =\int_{-4}^{-1} \int_{\mathbb{R}^{N}}\left(\partial_{r} w_{r}^{\varepsilon}\right)^{2} \frac{r G(x,-t)}{-t} d x d t+I+I I+I I I .
\end{aligned}
$$

Now,

$$
\partial_{r} w_{r}^{\varepsilon}=-\frac{w^{\varepsilon}\left(r x, r^{2} t\right)}{r^{2}}+\frac{\nabla w^{\varepsilon}\left(r x, r^{2} t\right)}{r} \cdot x+2 t w_{t}^{\varepsilon}\left(r x, r^{2} t\right) .
$$

There holds,

$$
\left|w^{\varepsilon}\left(r x, r^{2} t\right)\right| \leq\left|w^{\varepsilon}(0,0)\right|+\left(A_{1}+A_{2}\right) A_{4}\left(|x|+|t|^{1 / 2}\right) r,
$$

and for $0<r<1$,

$$
\begin{aligned}
& \left|\left(-\frac{w^{\varepsilon}\left(r x, r^{2} t\right)}{r^{2}}+\frac{\nabla w^{\varepsilon}\left(r x, r^{2} t\right)}{r} \cdot x\right)\left(-2 \psi^{r} r f_{\varepsilon}^{r}\right) G(x,-t)\right| \leq \\
& \quad \leq\left(2 \frac{\left|u^{\varepsilon}(0,0)\right|}{r} A_{4}+4\left(A_{1}+A_{2}\right) A_{4}\left(|x|+|t|^{1 / 2}\right)\right)\left\|f_{\varepsilon}\right\|_{L^{\infty}} G(x,-t) .
\end{aligned}
$$


So that,

$$
\begin{gathered}
\left.\int_{-4}^{-1} \int_{\mathbb{R}^{N}}\left(-\frac{w^{\varepsilon}\left(r x, r^{2} t\right)}{r^{2}}+\frac{\nabla w^{\varepsilon}\left(r x, r^{2} t\right)}{r} \cdot x\right)\left(-2 \psi^{r} r f_{\varepsilon}^{r}\right)\right) G(x,-t) d x d t \geq \\
\geq-C_{2}\left\|f_{\varepsilon}\right\|_{L^{\infty}} \frac{\left|u^{\varepsilon}(0,0)\right|}{r}-\bar{C}_{1}\left\|f_{\varepsilon}\right\|_{L^{\infty}} .
\end{gathered}
$$

Now,

$$
\begin{aligned}
& \left|\int_{-4}^{-1} \int_{\mathbb{R}^{N}}\left(2 t w_{t}^{\varepsilon}\left(r x, r^{2} t\right)\left(-2 \psi^{r} r f_{\varepsilon}^{r}\right)\right) G(x,-t) d x d t\right| \leq \\
& \quad \leq 16\left\|f_{\varepsilon}\right\|_{L^{\infty}\left(B_{R}(0) \times(-4,0)\right)} \int_{-4}^{-1} \int_{\mathbb{R}^{N}}\left|w_{t}^{\varepsilon}\left(r x, r^{2} t\right)\right| \psi^{r} r G(x,-t) d x d t= \\
& \quad=16\left\|f_{\varepsilon}\right\|_{L^{\infty}\left(B_{R}(0) \times(-4,0)\right)} \int_{-4}^{-1} \int_{\mathbb{R}^{N}}\left|\partial_{t}\left(u_{r}^{\varepsilon}\right)\right|\left(\psi^{r}\right)^{2} G(x,-t) d x d t \leq \\
& \quad \leq 32\left\|f_{\varepsilon}\right\|_{L^{\infty}\left(B_{R}(0) \times(-4,0)\right)}\left(\int_{-4}^{-1} \int_{\mathbb{R}^{N}}\left|\partial_{t}\left(u_{r}^{\varepsilon}\right)\right|^{2}\left(\psi^{r}\right)^{2} G(x,-t) d x d t\right)^{1 / 2} .
\end{aligned}
$$

Now, let $v=u_{r}^{\varepsilon}$. Then $v$ is a solution to $\Delta v-v_{t}=\beta_{\varepsilon / r}(v)+r f_{\varepsilon}{ }^{r}$. Thus,

$$
\begin{aligned}
\int_{-4}^{-1} & \int_{\mathbb{R}^{N}} v_{t}^{2}\left(\psi^{r}\right)^{2} G(x,-t) d x d t=\int_{-4}^{-1} \int_{\mathbb{R}^{N}} v_{t} \Delta v\left(\psi^{r}\right)^{2} G(x,-t) d x d t- \\
& -\int_{-4}^{-1} \int_{\mathbb{R}^{N}} \beta_{\varepsilon / r}(v) v_{t}\left(\psi^{r}\right)^{2} G(x,-t) d x d t-\int_{-4}^{-1} \int_{\mathbb{R}^{N}} r f_{\varepsilon}^{r} v_{t}\left(\psi^{r}\right)^{2} G(x,-t) d x d t= \\
& =i)+i i)+i i i) .
\end{aligned}
$$

There holds,

$$
\begin{aligned}
i)=- & \int_{-4}^{-1} \int_{\mathbb{R}^{N}} \nabla v_{t} \nabla v\left(\psi^{r}\right)^{2} G(x,-t) d x d t-2 \int_{-4}^{-1} \int_{\mathbb{R}^{N}} v_{t} \nabla v \psi^{r} \nabla \psi^{r} G(x,-t) d x d t- \\
& -\int_{-4}^{-1} \int_{\mathbb{R}^{N}} v_{t} \nabla v\left(\psi^{r}\right)^{2} \nabla G d x d t .
\end{aligned}
$$

Since for $x \in \mathbb{R}^{N},-4 \leq t \leq-1,|\nabla G(x,-t)|=\left|\frac{x}{2 t} G(x,-t)\right| \leq C G(x,-t)^{1 / 2} G(x,-2 t)^{1 / 2}$, $\left|G_{t}(x,-t)\right| \leq C G(x,-2 t)$ and $|\nabla v(x, t)|=\left|\nabla u^{\varepsilon}\left(r x, r^{2} t\right)\right| \leq A_{2}$ in the support of $\psi^{r}$, there holds

$$
\begin{aligned}
i) \leq\left.\int_{\mathbb{R}^{N}}|\nabla v|^{2}\left(\psi^{r}\right)^{2} G(x,-t) d x\right|_{t=-4} ^{t=-1}+\int_{-4}^{-1} \int_{\mathbb{R}^{N}}|\nabla v|^{2}\left(\psi^{r}\right)^{2}\left|G_{t}\right| d x d t \\
+2\left(\int_{-4}^{-1} \int_{\mathbb{R}^{N}} v_{t}^{2}\left(\psi^{r}\right)^{2} G(x,-t) d x d t\right)^{1 / 2}\left(\int_{-4}^{-1} \int_{\mathbb{R}^{N}}|\nabla v|^{2}\left|\nabla \psi^{r}\right|^{2} G(x,-t) d x d t\right)^{1 / 2}+ \\
+C\left(\int_{-4}^{-1} \int_{\mathbb{R}^{N}} v_{t}^{2}\left(\psi^{r}\right)^{2} G(x,-t) d x d t\right)^{1 / 2}\left(\int_{-4}^{-1} \int_{\mathbb{R}^{N}}|\nabla v|^{2}\left(\psi^{r}\right)^{2} G(x,-2 t) d x d t\right)^{1 / 2} \leq \\
\leq \eta \int_{-4}^{-1} \int_{\mathbb{R}^{N}} v_{t}^{2}\left(\psi^{r}\right)^{2} G(x,-t) d x d t+C_{\eta}\left(A_{2}, A_{4}\right)
\end{aligned}
$$

for $r \leq 1$. 
Proceeding in a similar way,

$$
\text { iii }) \leq \eta \int_{-4}^{-1} \int_{\mathbb{R}^{N}} v_{t}^{2}\left(\psi^{r}\right)^{2} G(x,-t) d x d t+C_{\eta}\left\|f_{\varepsilon}\right\|_{L^{\infty}\left(B_{R}(0) \times(-4,0)\right)}
$$

for $r \leq 1$.

Using that $\beta_{\varepsilon / r}(v) v_{t}=\partial_{t} B_{\varepsilon / r}(v)$ and $0 \leq B_{\varepsilon / r}(s) \leq M$ we get for ii)

$$
\text { ii) } \leq C(M) \text {. }
$$

Thus,

$$
\left|\int_{-4}^{-1} \int_{\mathbb{R}^{N}}\left(\partial_{t} u_{r}^{\varepsilon}\right)^{2}\left(\psi^{r}\right)^{2} G(x,-t) d x d t\right| \leq C\left(A_{2}, A_{4}, M\right)+C\left\|f_{\varepsilon}\right\|_{L^{\infty}\left(B_{R}(0) \times(-4,0)\right)} .
$$

Summing up,

$$
I \geq-C_{2}\left\|f_{\varepsilon}\right\|_{L^{\infty}\left(B_{R}(0) \times(-4,0)\right)} \frac{\left|u^{\varepsilon}(0,0)\right|}{r}-C_{1}\left(\left\|f_{\varepsilon}\right\|_{L^{\infty}\left(B_{R}(0) \times(-4,0)\right)}+\left\|f_{\varepsilon}\right\|_{L^{\infty}\left(B_{R}(0) \times(-4,0)\right)}^{3 / 2}\right) .
$$

Since $\psi \equiv 1$ in $B_{R / 2}(0)$,

$$
\begin{aligned}
I I \geq & -C\left(A_{1}, A_{2}, A_{4}\right)\left\{\frac{1}{r}\|G\|_{L^{1}((|x|>R / 2 r) \times(1,4))}+\||x| G\|_{L^{1}((|x|>R / 2 r) \times(1,4))}\right. \\
& \left.+\frac{1}{r^{\frac{N}{2}}}\left\|u_{t}^{\varepsilon}\right\|_{L^{2}\left(B_{R}(0) \times(-4,0)\right)}\|G\|_{L^{2}((|x|>R / 2 r) \times(1,4))}\right\} \geq-\bar{C}_{3} C(R) e^{-\bar{C}_{4} \frac{R^{2}}{r^{2}}}
\end{aligned}
$$

where $C(R)=\max \left\{R^{-1}, R^{-N / 2}\right\}$.

Since $0 \leq s \beta_{\varepsilon}(s) \leq M_{1}, 0 \leq B_{\varepsilon}(s) \leq M$ and $\psi \equiv 1$ in $B_{R / 2}(0)$,

$$
I I+I I I \geq-C_{3} C(R) e^{-C_{4} \frac{R^{2}}{r^{2}}} .
$$

The theorem is proved.

By passing to the limit as $\varepsilon$ goes to 0 we get a monotonicity formula for limits $u$ of solutions $u^{\varepsilon}$ to $P_{\varepsilon}\left(f_{\varepsilon}\right)$. Moreover, we prove that - when $u(0,0)=0$ - the functional $\mathcal{W}(r)$ has a limit as $r \rightarrow 0^{+}$.

Theorem 2.2 (Monotonicity Formula). Let $u^{\varepsilon}, f_{\varepsilon}$ and $\psi$ as in Theorem 2.1. Let $u=$ $\lim _{\varepsilon \rightarrow 0} u^{\varepsilon}$ uniformly on compact subsets of $\mathcal{D}, \chi=\lim _{\varepsilon \rightarrow 0} B_{\varepsilon}\left(u^{\varepsilon}\right) *$-weakly in $L^{\infty}(\mathcal{D})$. Let $A \geq\left\|f_{\varepsilon}\right\|_{L^{\infty}\left(B_{R}(0) \times(-4,0)\right)}$ and, for $0<r \leq 1$,

$$
\begin{aligned}
\mathcal{W}(r)=\mathcal{W}(r, u, \psi, \chi) & \\
& =\frac{1}{r^{2}} \int_{-4 r^{2}}^{-r^{2}} \int_{\mathbb{R}^{N}}\left(|\nabla(u \psi)|^{2}+2 \psi^{2} \chi+\frac{1}{2} \frac{(u \psi)^{2}}{t}\right) G(x,-t) d x d t .
\end{aligned}
$$

Then, for $1 \geq \rho_{1}>\rho_{2}>0$

$$
\begin{aligned}
\mathcal{W}\left(\rho_{1}\right) & -\mathcal{W}\left(\rho_{2}\right) \geq \int_{\rho_{2}}^{\rho_{1}} \int_{-4}^{-1} \int_{\mathbb{R}^{N}}\left(\partial_{r} w_{r}\right)^{2} \frac{r G(x,-t)}{-t} d x d t d r- \\
& -C_{1}\left(A+A^{3 / 2}\right)\left(\rho_{1}-\rho_{2}\right)-C_{2} A|u(0,0)| \log \left(\frac{\rho_{1}}{\rho_{2}}\right)-C_{3} C(R) \int_{\rho_{2}}^{\rho_{1}} e^{-C_{4} \frac{R^{2}}{r^{2}}} d r,
\end{aligned}
$$

where $C_{i}$ and $C(R)$ are as in Theorem 2.1 and $w(x, t)=\psi(x) u(x, t)$. In particular, if $u(0,0)=0$, there exists 


$$
\delta:=\lim _{r \rightarrow 0^{+}} \mathcal{W}(r)>-\infty
$$

Proof. Integration of (2.2) gives

$$
\begin{aligned}
\mathcal{W}^{\varepsilon}\left(\rho_{1}, u^{\varepsilon}, \psi\right) & -\mathcal{W}^{\varepsilon}\left(\rho_{2}, u^{\varepsilon}, \psi\right) \geq \int_{\rho_{2}}^{\rho_{1}} \int_{-4}^{-1} \int_{\mathbb{R}^{N}}\left(\partial_{r} w_{r}^{\varepsilon}\right)^{2} \frac{r G(x,-t)}{-t} d x d t d r- \\
& -C_{1}\left(A+A^{3 / 2}\right)\left(\rho_{1}-\rho_{2}\right)-C_{2} A\left|u^{\varepsilon}(0,0)\right| \log \left(\frac{\rho_{1}}{\rho_{2}}\right)-C_{3} C(R) \int_{\rho_{2}}^{\rho_{1}} e^{-C_{4} \frac{R^{2}}{r^{2}}} d r
\end{aligned}
$$

and the result follows by passing to the limit. In fact,

$$
\mathcal{W}^{\varepsilon}\left(r, u^{\varepsilon}, \psi\right) \rightarrow \mathcal{W}(r, u, \psi, \chi)
$$

since $u^{\varepsilon} \rightarrow u$ and $\nabla u^{\varepsilon} \rightarrow \nabla u$ almost everywhere and $\left|u^{\varepsilon}\right| \leq A_{1},\left|\nabla u^{\varepsilon}\right| \leq A_{2}$ on the support of $\psi$. We also use that $B_{\varepsilon}\left(u^{\varepsilon}\right) \rightarrow \chi *$-weakly in $L_{l o c}^{\infty}$.

On the other hand, for every $0<\rho_{2}<r<\rho_{1} \leq 1$,

$$
\liminf _{\varepsilon \rightarrow 0} \int_{-4}^{-1} \int_{\mathbb{R}^{N}}\left(\partial_{r} w_{r}^{\varepsilon}\right)^{2} \frac{r G(x,-t)}{-t} d x d t \geq \int_{-4}^{-1} \int_{\mathbb{R}^{N}}\left(\partial_{r} w_{r}\right)^{2} \frac{r G(x,-t)}{-t} d x d t .
$$

In fact,

$$
\begin{aligned}
\partial_{r} w_{r}^{\varepsilon}= & -\frac{u^{\varepsilon}\left(r x, r^{2} t\right) \psi(r x)}{r^{2}}+\frac{\psi(r x) \nabla u^{\varepsilon}\left(r x, r^{2} t\right) \cdot x}{r}+ \\
& +\frac{u^{\varepsilon}\left(r x, r^{2} t\right) \nabla \psi(r x) \cdot x}{r}+2 t \psi(r x) \partial_{t} u^{\varepsilon}\left(r x, r^{2} t\right) .
\end{aligned}
$$

We use the convergence and boundedness of $u^{\varepsilon}$ and $\nabla u^{\varepsilon}$ stated above and the weak convergence of $\partial_{t} u^{\varepsilon}$ to $\partial_{t} u$ in $L^{2}$ on compact sets together with estimate (2.8) that implies that

$$
\psi(r x) \partial_{t} u^{\varepsilon}\left(r x, r^{2} t\right) \rightarrow \psi(r x) \partial_{t} u\left(r x, r^{2} t\right)
$$

weakly in $L^{2}\left(\mathbb{R}^{N} \times(-4,-1),(-t) G(x,-t) d x d t\right)$. Also, (2.8) gives uniform bounds that allow to pass to the limit in the triple integral to get

$$
\begin{aligned}
\liminf _{\varepsilon \rightarrow 0} \int_{\rho_{2}}^{\rho_{1}} \int_{-4}^{-1} \int_{\mathbb{R}^{N}}\left(\partial_{r} w_{r}^{\varepsilon}\right)^{2} \frac{r G(x,-t)}{-t} d x d t d r \geq \\
\geq \int_{\rho_{2}}^{\rho_{1}} \int_{-4}^{-1} \int_{\mathbb{R}^{N}}\left(\partial_{r} w_{r}\right)^{2} \frac{r G(x,-t)}{-t} d x d t d r
\end{aligned}
$$

and this proves the theorem.

Now, we prove that any blow up limit of a function $u=\lim u^{\varepsilon}$ at a point where $u(0,0)=0$ verifies that the global functional of Weiss (cf. [18]) is constant with a constant independent of the blow up sequence and we prove that the blow up limit is homogeneous.

Corollary 2.1. Let $u$ and $\chi$ as in Theorem 2.2. Assume $(0,0) \in \mathcal{D} \cap \partial\{u>0\}$. Let $u_{\lambda}(x, t)=$ $\frac{1}{\lambda} u\left(\lambda x, \lambda^{2} t\right), \chi^{\lambda}(x, t)=\chi\left(\lambda x, \lambda^{2} t\right)$. Let $u_{0}=\lim _{j \rightarrow \infty} u_{\lambda_{j}}$ uniformly on compact sets of $\mathbb{R}^{N} \times$ $(-\infty, 0], \chi_{0}=\lim _{j \rightarrow \infty} \chi^{\lambda_{j}}$ *-weakly in $L^{\infty}$ on compact sets of $\mathbb{R}^{N} \times(-\infty, 0]$, with $\lambda_{j} \rightarrow 0^{+}$.

Then, for $r>0$

$$
\begin{aligned}
& \mathcal{W}_{0}(r)=\mathcal{W}\left(r, u_{0}, 1, \chi_{0}\right) \\
& =\frac{1}{r^{2}} \int_{-4 r^{2}}^{-r^{2}} \int_{\mathbb{R}^{N}}\left(\left|\nabla u_{0}\right|^{2}+2 \chi_{0}+\frac{1}{2} \frac{u_{0}^{2}}{t}\right) G(x,-t) d x d t \equiv \delta
\end{aligned}
$$


where $\delta$ is the constant in Theorem 2.2. In particular, $\delta$ is independent of the sequence $\lambda_{j}$ and of the cut off function $\psi$. Moreover, for $\rho_{1} \geq \rho_{2}>0$

$$
0=\mathcal{W}_{0}\left(\rho_{1}\right)-\mathcal{W}_{0}\left(\rho_{2}\right) \geq \int_{\rho_{2}}^{\rho_{1}} \int_{-4}^{-1} \int_{\mathbb{R}^{N}}\left(\partial_{r}\left(u_{0}\right)_{r}\right)^{2} \frac{r G(x,-t)}{-t} d x d t d r
$$

Therefore, $u_{0}$ is homogeneous in $\mathbb{R}^{N} \times(-\infty, 0)$. This is, for every $x \in \mathbb{R}^{N}, t<0$,

$$
u_{0}\left(r x, r^{2} t\right)=r u_{0}(x, t) \quad \text { for } \quad r>0 .
$$

Proof. Let $r>0$. By Theorem 2.2 if $\psi \in C_{0}^{\infty}\left(B_{R}(0)\right), \psi \equiv 1$ in $B_{R / 2}(0), 0 \leq \psi \leq 1$,

$$
\mathcal{W}(\lambda r, u, \psi, \chi) \rightarrow \delta \quad \text { as } \quad \lambda \rightarrow 0
$$

On the other hand,

$$
\begin{aligned}
\mathcal{W}\left(\lambda_{j} r\right. & , u, \psi, \chi)=\int_{-4}^{-1} \int_{\mathbb{R}^{N}}\left(\left|\nabla(u \psi)_{\lambda_{j} r}\right|^{2}+2\left(\psi^{2} \chi\right)^{\lambda_{j} r}+\frac{1}{2} \frac{(u \psi)_{\lambda_{j} r}^{2}}{t}\right) G(x,-t) d x d t= \\
& =\int_{-4}^{-1} \int_{\mathbb{R}^{N}}\left(\left|\nabla\left(u_{\lambda_{j}} \psi^{\lambda_{j}}\right)_{r}\right|^{2}+2\left(\left(\psi^{\lambda_{j}}\right)^{2} \chi^{\lambda_{j}}\right)^{r}+\frac{1}{2} \frac{\left(u_{\lambda_{j}} \psi^{\lambda_{j}}\right)_{r}^{2}}{t}\right) G(x,-t) d x d t= \\
& =\frac{1}{r^{2}} \int_{-4 r^{2}}^{-r^{2}} \int_{\mathbb{R}^{N}}\left(\left|\nabla\left(u_{\lambda_{j}} \psi^{\lambda_{j}}\right)\right|^{2}+2\left(\psi^{\lambda_{j}}\right)^{2} \chi^{\lambda_{j}}+\frac{1}{2} \frac{\left(u_{\lambda_{j}} \psi^{\lambda_{j}}\right)^{2}}{t}\right) G(x,-t) d x d t= \\
& =\mathcal{W}\left(r, u_{\lambda_{j}}, \psi^{\lambda_{j}}, \chi^{\lambda_{j}}\right) .
\end{aligned}
$$

Now, there holds that $u_{\lambda_{j}} \rightarrow u_{0}$ as $j \rightarrow \infty$ uniformly on compact sets of $\mathbb{R}^{N} \times(-\infty, 0]$, $\nabla u_{\lambda_{j}} \rightarrow \nabla u_{0}$ almost everywhere in $\mathbb{R}^{N} \times(-\infty, 0]$ and $\left|u_{\lambda_{j}}\right| \leq A_{1},\left|\nabla u_{\lambda_{j}}\right| \leq A_{2}$ on the support of $\psi^{\lambda_{j}}$ where $A_{i}$ are defined in Theorem 2.1.

On the other hand, $\psi^{\lambda_{j}} \rightarrow 1$ almost everywhere, $\nabla \psi^{\lambda_{j}} \rightarrow 0$ almost everywhere with $\left|\nabla \psi^{\lambda_{j}}\right| \leq$ $A_{4}$ for $\lambda_{j} \leq 1$. Therefore,

$$
\begin{aligned}
\frac{1}{r^{2}} \int_{-4 r^{2}}^{-r^{2}} \int_{\mathbb{R}^{N}} & \left(\left|\nabla\left(u_{\lambda_{j}} \psi^{\lambda_{j}}\right)\right|^{2}+\frac{1}{2} \frac{\left(u_{\lambda_{j}} \psi^{\lambda_{j}}\right)^{2}}{t}\right) G(x,-t) d x d t \rightarrow \\
& \rightarrow \frac{1}{r^{2}} \int_{-4 r^{2}}^{-r^{2}} \int_{\mathbb{R}^{N}}\left(\left|\nabla u_{0}\right|^{2}+\frac{1}{2} \frac{u_{0}^{2}}{t}\right) G(x,-t) d x d t \quad \text { as } \quad j \rightarrow \infty .
\end{aligned}
$$

Moreover, $\chi^{\lambda_{j}} \rightarrow \chi_{0}$ *-weakly in $L^{\infty}$ on compact sets of $\mathbb{R}^{N} \times(-\infty, 0]$. Thus,

$$
\frac{1}{r^{2}} \int_{-4 r^{2}}^{-r^{2}} \int_{\mathbb{R}^{N}}\left(\psi^{\lambda_{j}}\right)^{2} \chi^{\lambda_{j}} G(x,-t) d x d t \rightarrow \frac{1}{r^{2}} \int_{-4 r^{2}}^{-r^{2}} \int_{\mathbb{R}^{N}} \chi_{0} G(x,-t) d x d t .
$$


In fact, let $\varepsilon>0$ and let $K>0$ be such that $\frac{1}{r^{2}} \int_{-4 r^{2}}^{-r^{2}} \int_{|x|>K} M G(x,-t) d x d t<\frac{\varepsilon}{4}$. Then,

$$
\begin{aligned}
& \left|\frac{1}{r^{2}} \int_{-4 r^{2}}^{-r^{2}} \int_{\mathbb{R}^{N}}\left\{\left(\psi^{\lambda_{j}}\right)^{2} \chi^{\lambda_{j}}-\chi_{0}\right\} G(x,-t) d x d t\right| \leq \\
& \leq\left|\frac{1}{r^{2}} \int_{-4 r^{2}}^{-r^{2}} \int_{|x|<K}\left\{\left(\psi^{\lambda_{j}}\right)^{2} \chi^{\lambda_{j}}-\chi_{0}\right\} G(x,-t) d x d t\right|+\frac{\varepsilon}{2}= \\
& \quad=\left|\frac{1}{r^{2}} \int_{-4 r^{2}}^{-r^{2}} \int_{|x|<K}\left\{\chi^{\lambda_{j}}-\chi_{0}\right\} G(x,-t) d x d t\right|+\frac{\varepsilon}{2}<\varepsilon
\end{aligned}
$$

if $j$ is sufficiently large. Thus, (2.13) holds. Therefore, for every $r>0$,

$$
\mathcal{W}\left(r, u_{0}, 1, \chi_{0}\right)=\lim _{j \rightarrow \infty} \mathcal{W}\left(r, u_{\lambda_{j}}, \psi^{\lambda_{j}}, \chi^{\lambda_{j}}\right)=\lim _{j \rightarrow \infty} \mathcal{W}\left(\lambda_{j} r, u, \psi, \chi\right) \equiv \delta .
$$

On the other hand, if $0<\rho_{2} \leq \rho_{1}$, by Theorem 2.2,

$$
\begin{aligned}
& 0=\mathcal{W}\left(\rho_{1}, u_{0}, 1, \chi_{0}\right)-\mathcal{W}\left(\rho_{2}, u_{0}, 1, \chi_{0}\right)=\lim _{j \rightarrow \infty}\left\{\mathcal{W}\left(\lambda_{j} \rho_{1}, u, \psi, \chi\right)-\mathcal{W}\left(\lambda_{j} \rho_{2}, u, \psi, \chi\right)\right\} \geq \\
& \geq \liminf _{j \rightarrow \infty}\left[\int_{\rho_{2}}^{\rho_{1}} \int_{-4}^{-1} \int_{\mathbb{R}^{N}}\left(\partial_{r}\left(w_{\lambda_{j}}\right)_{r}\right)^{2} \frac{r G(x,-t)}{-t} d x d t d r-\right. \\
& \left.-C_{1}\left(A+A^{3 / 2}\right) \lambda_{j}\left(\rho_{1}-\rho_{2}\right)-C_{3} C(R) \lambda_{j} \int_{\rho_{2}}^{\rho_{1}} e^{-C_{4} \frac{R^{2}}{\lambda_{j}^{2} r^{2}}} d r\right]= \\
& =\liminf _{j \rightarrow \infty} \int_{\rho_{2}}^{\rho_{1}} \frac{1}{r^{4}} \int_{-4 r^{2}}^{-r^{2}} \int_{\mathbb{R}^{N}}\left(-u_{\lambda_{j}} \psi^{\lambda_{j}}+\psi^{\lambda_{j}} \nabla u_{\lambda_{j}} \cdot x+u_{\lambda_{j}} \nabla \psi^{\lambda_{j}} \cdot x+\right. \\
& \left.+2 t \psi^{\lambda_{j}} \partial_{t} u_{\lambda_{j}}\right)^{2} \frac{r G(x,-t)}{-t} d x d t d r= \\
& =\liminf _{j \rightarrow \infty}\left\{\int_{\rho_{2}}^{\rho_{1}} \frac{1}{r^{4}} \int_{-4 r^{2}}^{-r^{2}} \int_{\mathbb{R}^{N}}\left(-u_{\lambda_{j}} \psi^{\lambda_{j}}+\psi^{\lambda_{j}} \nabla u_{\lambda_{j}} \cdot x+u_{\lambda_{j}} \nabla \psi^{\lambda_{j}} \cdot x\right)^{2} \frac{r G(x,-t)}{-t} d x d t d r+\right. \\
& +\int_{\rho_{2}}^{\rho_{1}} \frac{1}{r^{4}} \int_{-4 r^{2}}^{-r^{2}} \int_{\mathbb{R}^{N}}\left(2 t \psi^{\lambda_{j}} \partial_{t} u_{\lambda_{j}}\right)^{2} \frac{r G(x,-t)}{-t} d x d t d r+ \\
& +\int_{\rho_{2}}^{\rho_{1}} \frac{2}{r^{4}} \int_{-4 r^{2}}^{-r^{2}} \int_{\mathbb{R}^{N}}\left(-u_{\lambda_{j}} \psi^{\lambda_{j}}+\psi^{\lambda_{j}} \nabla u_{\lambda_{j}} \cdot x+u_{\lambda_{j}} \nabla \psi^{\lambda_{j}} \cdot x\right) \\
& \left.\left(2 t \psi^{\lambda_{j}} \partial_{t} u_{\lambda_{j}}\right) \frac{r G(x,-t)}{-t} d x d t d r\right\}= \\
& =\liminf _{j \rightarrow \infty}\left\{\int_{\rho_{2}}^{\rho_{1}} I d r+\int_{\rho_{2}}^{\rho_{1}} I I d r+\int_{\rho_{2}}^{\rho_{1}} I I I d r\right\} .
\end{aligned}
$$

It is easy to see that for every $0<\rho_{2}<r<\rho_{1}$ and $j$ sufficiently large

$$
|I|=\left|\frac{1}{r^{4}} \int_{-4 r^{2}}^{-r^{2}} \int_{\mathbb{R}^{N}}\left(-u_{\lambda_{j}} \psi^{\lambda_{j}}+\psi^{\lambda_{j}} \nabla u_{\lambda_{j}} \cdot x+u_{\lambda_{j}} \nabla \psi^{\lambda_{j}} \cdot x\right)^{2} \frac{r G(x,-t)}{-t} d x d t\right| \leq C
$$

and moreover,

$$
I \rightarrow \frac{1}{r^{4}} \int_{-4 r^{2}}^{-r^{2}} \int_{\mathbb{R}^{N}}\left(-u_{0}+\nabla u_{0} \cdot x\right)^{2} \frac{r G(x,-t)}{-t} d x d t
$$


On the other hand, by (2.8) we get for $0<\rho_{2}<r<\rho_{1}$ and $\varepsilon<\varepsilon_{0}(\lambda)$,

$$
\left|\int_{-4 r^{2}}^{-r^{2}} \int_{\mathbb{R}^{N}}\left(\partial_{t} u_{\lambda}^{\varepsilon}\right)^{2}\left(\psi^{\lambda}\right)^{2} G(x,-t) d x d t\right| \leq C\left(A_{2}, A_{4}, M, A, \rho_{1}, \rho_{2}\right) .
$$

Thus, since $\partial_{t} u_{\lambda_{j}}^{\varepsilon} \rightarrow \partial_{t} u_{\lambda_{j}}$ as $\varepsilon \rightarrow 0$ weakly in $L^{2}$ on compact sets,

$$
\left|\int_{-4 r^{2}}^{-r^{2}} \int_{\mathbb{R}^{N}}\left(\partial_{t} u_{\lambda_{j}}\right)^{2}\left(\psi^{\lambda_{j}}\right)^{2} G(x,-t) d x d t\right| \leq C\left(A_{2}, A_{4}, M, A, \rho_{1}, \rho_{2}\right) .
$$

Therefore,

$$
|I I| \leq \tilde{C}\left(A_{2}, A_{4}, M, A, \rho_{1}, \rho_{2}\right) .
$$

Thus, for a subsequence that we still call $\lambda_{j}$,

$$
\psi^{\lambda_{j}} \partial_{t} u_{\lambda_{j}}=\partial_{t}\left(\psi^{\lambda_{j}} u_{\lambda_{j}}\right) \rightarrow \partial_{t} u_{0} \quad \text { as } \quad j \rightarrow \infty
$$

weakly in $L^{2}\left(\mathbb{R}^{N} \times\left(-4 r^{2},-r^{2}\right) ;(-t) G(x,-t) d x d t\right)$.

In fact, the estimate (2.15) implies that $\psi^{\lambda_{j}} \partial_{t} u_{\lambda_{j}}$ converges weakly to a function $v_{0}$ in $L^{2}\left(\mathbb{R}^{N} \times\right.$ $\left.\left(-4 r^{2},-r^{2}\right) ;(-t) G(x,-t) d x d t\right)$. Since $\partial_{t} u_{\lambda_{j}}$ converges to $\partial_{t} u_{0}$ weakly in $L_{l o c}^{2}\left(\mathbb{R}^{N} \times(-\infty, 0)\right)$, and $\psi^{\lambda_{j}} \equiv 1$ in $|x|<\frac{1}{2 \lambda_{j}}$, we get $(2.16)$.

Now, (2.15) and (2.16) imply that

$$
\begin{aligned}
\liminf _{j \rightarrow \infty} \frac{1}{r^{4}} \int_{-4 r^{2}}^{-r^{2}} \int_{\mathbb{R}^{N}}\left(2 t \psi^{\lambda_{j}} \partial_{t} u_{\lambda_{j}}\right)^{2} \frac{r G(x,-t)}{-t} d x d t \geq \\
\geq \frac{1}{r^{4}} \int_{-4 r^{2}}^{-r^{2}} \int_{\mathbb{R}^{N}}\left(2 t \partial_{t} u_{0}\right)^{2} \frac{r G(x,-t)}{-t} d x d t
\end{aligned}
$$

and

$$
I I I \rightarrow \frac{2}{r^{2}} \int_{-4 r^{2}}^{-r^{2}} \int_{\mathbb{R}^{N}}\left(-u_{0}+\nabla u_{0} \cdot x\right)\left(2 t \partial_{t} u_{0}\right) \frac{r G(x,-t)}{-t} d x d t
$$

Moreover,

$$
|I I I| \leq C
$$

with $C$ independent of $\lambda$ and $r$, for $\lambda$ small enough and $0<\rho_{2}<r<\rho_{1}$.

Finally, the uniform estimates and the convergence (2.14), (2.17) and (2.18) imply that

$$
\begin{gathered}
0=\mathcal{W}\left(\rho_{1}, u_{0}, 1, \chi_{0}\right)-\mathcal{W}\left(\rho_{2}, u_{0}, 1, \chi_{0}\right)=\lim _{j \rightarrow \infty}\left\{\mathcal{W}\left(\lambda_{j} \rho_{1}, u, \psi, \chi\right)-\mathcal{W}\left(\lambda_{j} \rho_{2}, u, \psi, \chi\right)\right\} \geq \\
\geq \int_{\rho_{2}}^{\rho_{1}} \int_{-4}^{-1} \int_{\mathbb{R}^{N}}\left(\partial_{r}\left(u_{0}\right)_{r}\right)^{2} \frac{r G(x,-t)}{-t} d x d t d r \geq 0 .
\end{gathered}
$$

Thus, for every $r>0,-4 \leq t \leq-1$,

$$
u_{0}\left(r x, r^{2} t\right)=r u_{0}(x, t)
$$

In particular, for $t=-1$,

Therefore, for any $t<0, y \in \mathbb{R}^{N}$,

$$
u_{0}\left(r x,-r^{2}\right)=r u_{0}(x,-1)=r H(x) .
$$

$$
u_{0}(y, t)=u_{0}\left(r x,-r^{2}\right)=\sqrt{-t} H\left(\frac{y}{\sqrt{-t}}\right),
$$


and

$$
u_{0}\left(r y, r^{2} t\right)=r u_{0}(y, t)
$$

for every $r>0, y \in \mathbb{R}^{N}, t<0$.

The corollary is proved.

Proposition 2.1. Let $u, \chi, \psi$ as in Theorem 2.2. Assume $(0,0) \in \mathcal{D} \cap \partial\{u>0\}$ and let $\delta$ as in Theorem 2.2. Then,

$$
\delta=\lim _{r \rightarrow 0} \frac{1}{r^{2}} \int_{-4 r^{2}}^{-r^{2}} \int_{\mathbb{R}^{N}} 2 \psi^{2} \chi G(x,-t) d x d t
$$

Proof. In Theorem 2.2 we proved that

$$
\delta=\lim _{r \rightarrow 0} \frac{1}{r^{2}} \int_{-4 r^{2}}^{-r^{2}} \int_{\mathbb{R}^{N}}\left\{|\nabla(u \psi)|^{2}+2 \psi^{2} \chi+\frac{(u \psi)^{2}}{2 t}\right\} G(x,-t) d x d t .
$$

We claim that

$$
L:=\lim _{r \rightarrow 0} \int_{-4 r^{2}}^{-r^{2}} \frac{1}{r^{2}} \int_{\mathbb{R}^{N}}\left\{|\nabla(u \psi)|^{2}+\frac{(u \psi)^{2}}{2 t}\right\} G(x,-t) d x d t=0 .
$$

In fact, let $\lambda_{j} \rightarrow 0$ be a sequence such that $u_{\lambda_{j}}$ has a limit $u_{0}$. Then, for $r>0$ fixed

$$
\begin{aligned}
L & =\lim _{j \rightarrow \infty} \int_{-4 \lambda_{j}^{2} r^{2}}^{-\lambda_{j} r^{2}} \frac{1}{\lambda_{j}^{2} r^{2}} \int_{\mathbb{R}^{N}}\left\{|\nabla(u \psi)|^{2}+\frac{(u \psi)^{2}}{2 t}\right\} G(x,-t) d x d t= \\
& =\lim _{j \rightarrow \infty} \int_{-4 r^{2}}^{-r^{2}} \frac{1}{r^{2}} \int_{\mathbb{R}^{N}}\left\{\left|\nabla\left(u_{\lambda_{j}} \psi^{\lambda_{j}}\right)^{2}\right|+\frac{\left(u_{\lambda_{j}} \psi^{\lambda_{j}}\right)^{2}}{2 t}\right\} G(x,-t) d x d t= \\
& =\frac{1}{r^{2}} \int_{-4 r^{2}}^{-r^{2}} \int_{\mathbb{R}^{N}}\left(\left|\nabla u_{0}\right|^{2}+\frac{1}{2} \frac{u_{0}^{2}}{t}\right) G(x,-t) d x d t
\end{aligned}
$$

as proved in Corollary 2.1. Let us see that this last integral is zero. This is a consequence of the fact that $u_{0}$ is homogeneous and a solution to the homogeneous heat equation in the set $\left\{u_{0}>0\right\} \cup\{u \leq 0\}^{\circ}$. In fact, let $H=H(y)$ be such that $u_{0}(x, t)=\sqrt{-t} H\left(\frac{x}{\sqrt{-t}}\right)$. Then,

$$
\Delta H(y)=\frac{\partial}{\partial t} u_{0}(y,-1)=-\frac{1}{2} H(y)+\frac{1}{2} y \cdot \nabla H(y) .
$$

Thus,

$$
\begin{aligned}
& \frac{1}{r^{2}} \int_{-4 r^{2}}^{-r^{2}} \int_{\mathbb{R}^{N}}\left(\left|\nabla u_{0}\right|^{2}+\frac{1}{2} \frac{u_{0}^{2}}{t}\right) G(x,-t) d x d t= \\
& =\frac{3}{(2 \sqrt{\pi})^{N}} \int_{\mathbb{R}^{N}}\left\{|\nabla H(y)|^{2}-\frac{1}{2} H^{2}(y)\right\} e^{-\frac{1}{4}|y|^{2}} d y= \\
& =\frac{3}{(2 \sqrt{\pi})^{N}}\left\{-\int_{\mathbb{R}^{N}} H(y) \operatorname{div}\left(e^{-\frac{1}{4}|y|^{2}} \nabla H(y)\right) d y-\int_{\mathbb{R}^{N}} \frac{1}{2} H^{2}(y) e^{-\frac{1}{4}|y|^{2}} d y\right\}=0 .
\end{aligned}
$$


Corollary 2.2. Let $u, \chi, u_{0}$ and $\chi_{0}$ as in Corollary 2.1. Assume $(0,0) \in \mathcal{D} \cap \partial\{u>0\}$ and let $\delta$ as in Theorem 2.2. Then, for any $r>0$,

$$
\begin{aligned}
\delta & \equiv \mathcal{W}\left(r, u_{0}, 1, \chi_{0}\right) \\
& =\frac{1}{r^{2}} \int_{-4 r^{2}}^{-r^{2}} \int_{\mathbb{R}^{N}}\left(\left|\nabla u_{0}\right|^{2}+2 \chi_{0}+\frac{1}{2} \frac{u_{0}^{2}}{t}\right) G(x,-t) d x d t \\
& =\frac{1}{r^{2}} \int_{-4 r^{2}}^{-r^{2}} \int_{\mathbb{R}^{N}} 2 \chi_{0} G(x,-t) d x d t .
\end{aligned}
$$

In particular $0 \leq \delta \leq 6 M$.

Proof. The result follows from Corollary 2.1, the proof of Proposition 2.1 and the fact that $0 \leq \chi_{0} \leq M$.

Remark 2.2. Let $\mathcal{D} \subset \mathbb{R}^{N+1}$ be a domain. Let $u^{\varepsilon}$ be a family of uniformly bounded in $\operatorname{Lip}(1,1 / 2)$ norm - solutions to $P_{\varepsilon}\left(f_{\varepsilon}\right)$ in $\mathcal{D}$ with $f_{\varepsilon}$ uniformly bounded in $L^{\infty}(\mathcal{D})$. Let $u=\lim u^{\varepsilon}$ and $\chi=\lim B_{\varepsilon}\left(u^{\varepsilon}\right)$ in $\mathcal{D}$. Let $\left(x_{0}, t_{0}\right) \in \mathcal{D} \cap \partial\{u>0\}$. Then, if $\lambda_{0}$ is small enough, all the results of this section can be applied to the functions

$$
\begin{aligned}
& u^{\sigma}(x, t)=\frac{1}{\lambda_{0}} u^{\varepsilon}\left(x_{0}+\lambda_{0} x, t_{0}+\lambda_{0}^{2} t\right), \\
& \tilde{u}=\lim u^{\sigma} \\
& \tilde{\chi}=\lim B_{\sigma}\left(u^{\sigma}\right)
\end{aligned}
$$

where $\sigma=\varepsilon / \lambda_{0}$.

In fact, $u^{\sigma}$ are solutions to $P_{\sigma}\left(f_{\sigma}\right)$ with $f_{\sigma}(x, t)=\lambda_{0} f_{\varepsilon}\left(x_{0}+\lambda_{0} x, t_{0}+\lambda_{0}^{2} t\right)$. Also, $(0,0) \in$ $\tilde{\mathcal{D}} \cap \partial\{\tilde{u}>0\}$ and $B_{1}(0) \times[-4,0] \subset \tilde{\mathcal{D}}$ where $\tilde{\mathcal{D}}=\frac{1}{\lambda_{0}}\left(\mathcal{D}-\left(x_{0}, t_{0}\right)\right)$.

Remark 2.3. The value of $\delta\left(x_{0}, t_{0}\right)$ is closely related to the regularity of the boundary of $\{u>0\}$ in a neighborhood of $\left(x_{0}, t_{0}\right) \in \partial\{u>0\}$.

Indeed, let $u^{\varepsilon}$ and $f_{\varepsilon}$ as in Remark 2.2. Let $u=\lim u^{\varepsilon}, \chi=\lim B_{\varepsilon}\left(u^{\varepsilon}\right),\left(x_{0}, t_{0}\right) \in \partial\{u>0\}$ and $\chi_{0}(x, t)=\lim \chi\left(x_{0}+\lambda_{n} x, t_{0}+\lambda_{n}^{2} t\right)$, with $\lambda_{n} \rightarrow 0$. Assume that $\partial\{u>0\}$ is smooth in a neighborhood of $\left(x_{0}, t_{0}\right)$ with a nonhorizontal normal at $\left(x_{0}, t_{0}\right)$, and that $u^{+}$is nondegenerate at $\left(x_{0}, t_{0}\right)$ (i.e, $u^{+}$grows linearly from $\partial\{u>0\}$ ). Let $\psi$ be such that $\psi=\psi(x) \in C_{0}^{\infty}, 0 \leq \psi \leq 1$, $\psi \equiv 1$ in a neighborhood of $x_{0}$. Then

$$
\begin{aligned}
\delta\left(x_{0}, t_{0}\right) & =\lim _{r \rightarrow 0} \frac{1}{r^{2}} \int_{t_{0}-4 r^{2}}^{t_{0}-r^{2}} \int_{\mathbb{R}^{N}}\left(|\nabla(u \psi)|^{2}+2 \psi^{2} \chi+\frac{1}{2} \frac{(u \psi)^{2}}{t-t_{0}}\right) G\left(x-x_{0}, t_{0}-t\right) d x d t= \\
& =\int_{-4}^{-1} \int_{\mathbb{R}^{N}} 2 \chi_{0} G(x,-t) d x d t=3 M .
\end{aligned}
$$

In fact, let $u_{\lambda}(x, t)=\frac{1}{\lambda} u\left(x_{0}+\lambda x, t_{0}+\lambda^{2} t\right)$. Then, in some system of coordinates, $u_{\lambda}(x, t) \rightarrow$ $u_{0}(x, t)=\alpha x_{1}^{+}-\gamma x_{1}^{-}$with $\alpha>0$ and $\gamma \geq 0$.

Since for some sequence $\delta_{n}=\frac{\varepsilon_{j_{n}}}{\lambda_{n}} \rightarrow 0$ there holds that $\chi_{0}=\lim B_{\delta_{n}}\left(u^{\delta_{n}}\right)$ with $u^{\delta_{n}} \rightarrow u_{0}$ solutions to $P_{\delta_{n}}\left(f_{\delta_{n}}\right)$ uniformly bounded in $\operatorname{Lip}(1,1 / 2)$, with $f_{\delta_{n}} \rightarrow 0$ (see the proof of Theorem 3.1 ) and $\alpha>0$, there holds that $\chi_{0}=M$ in $\left\{x_{1}>0\right\}$.

If $\gamma>0$, then $\chi_{0}=0$ in $\left\{x_{1}<0\right\}$. Let us see that this is also true if $\gamma=0$. 
In fact, as in the proof of Proposition 5.1 in [13] we deduce that $\chi_{0}(x, t)=\chi_{0}(t) \in\{0, M\}$ in $\left\{x_{1}<0\right\}$ and $0<\frac{1}{2} \alpha^{2}=M-\chi_{0}(t)$ (see also Proposition 5.2 in [9]). Thus, $\chi_{0}(t) \equiv 0$ in $\left\{x_{1}<0\right\}$.

Replacing the value of $\chi_{0}$ in the formula of $\delta\left(x_{0}, t_{0}\right)$ we conclude that

$$
\delta\left(x_{0}, t_{0}\right)=3 M \text {. }
$$

Moreover, we prove in Theorem 9.7 in [14] that the regularity of the boundary of $\{u>0\}$ in a neighborhood of a boundary point is equivalent to $\delta$ being $3 M$ at that point, for the stationary case (see Section 4).

\section{Application I: Asymptotic developments}

In this section we apply the results of Section 2 to prove a result on asymptotic developments (Theorem 3.1). This result concerns points of $\partial\{u>0\}$ at which there is a one sided tangent ball. Note that these points are dense in $\partial\{u>0\}$. On the other hand, the knowledge of this type of asymptotic developments at points with a one sided tangent ball have proved to be an essential tool for the proof of the regularity of $\partial\{u>0\}$ in free boundary problems (see, for instance, [1], [4], [5]).

We point out that all the cases present in Theorem 3.1 appear in different situations, as the examples in [13] show.

We have

Theorem 3.1. Let $u^{\varepsilon_{j}}$ be a family of solutions to $P_{\varepsilon_{j}}\left(f_{\varepsilon_{j}}\right)$ in $\mathcal{D}$, uniformly bounded in Lip $(1,1 / 2)$ with $f_{\varepsilon_{j}}$ uniformly bounded in $L^{\infty}(\mathcal{D})$ and $\varepsilon_{j} \rightarrow 0$. Let $u=\lim u^{\varepsilon_{j}}$ in $\mathcal{D}$. Let $\left(x_{0}, t_{0}\right) \in \partial\{u>$ $0\} \cap \mathcal{D}$. Assume $\partial\{u>0\}$ has a nonhorizontal tangent ball $B$ at the point $\left(x_{0}, t_{0}\right)$. This is, there is a ball $B$ such that $\left(x_{0}, t_{0}\right) \in \partial B$, the normal to $B$ at $\left(x_{0}, t_{0}\right)$ has a nonzero spatial component and

$$
B \cap\left\{t \leq t_{0}\right\} \subset\{u>0\} \quad \text { or } B \cap\left\{t \leq t_{0}\right\} \subset\{u \leq 0\} .
$$

Let us call $\nu$ the interior unit spatial normal to $B$ at $\left(x_{0}, t_{0}\right)$ when $B \cap\left\{t \leq t_{0}\right\} \subset\{u>0\}$ and the exterior unit spatial normal to $B$ at $\left(x_{0}, t_{0}\right)$ when $B \cap\left\{t \leq t_{0}\right\} \subset\{u \leq 0\}$. Then, there exist $\alpha \geq 0$ and $\sigma \in \mathbb{R}$ such that

$$
u(x, t)=\alpha\left\langle x-x_{0}, \nu\right\rangle^{+}+\sigma\left\langle x-x_{0}, \nu\right\rangle^{-}+o\left(\left|x-x_{0}\right|+\left|t-t_{0}\right|^{1 / 2}\right) \quad \text { in } \quad\left\{t \leq t_{0}\right\} .
$$

Moreover, when $B \cap\left\{t \leq t_{0}\right\} \subset\{u>0\}$, there holds that

(1) If $\alpha>\sqrt{2 M}$, then $\sigma<0$ and $\alpha^{2}-\sigma^{2}=2 M$.

(2) If $\alpha=\sqrt{2 M}$, then $\sigma=\sqrt{2 M}$ or $\sigma=0$.

(3) If $0 \leq \alpha<\sqrt{2 M}$, then $\sigma=\alpha$.

When $B \cap\left\{t \leq t_{0}\right\} \subset\{u \leq 0\}$, the only possible cases are

(i) $\alpha \geq \sqrt{2 M}$ and $\sigma \leq 0$ with $\alpha^{2}-\sigma^{2}=2 M$.

(ii) $\alpha=\sigma=0$.

Proof. Without loss of generality we may assume that $\left(x_{0}, t_{0}\right)=(0,0)$ and $\nu=e_{1}$.

CASE I: Assume first that $B \cap\{t \leq 0\} \subset\{u>0\}$ and $e_{1}$ is the interior unit spatial normal to $B$ at $(0,0)$. By Lemma A.1 in [13] we know that there exists $\alpha \geq 0$ such that

$$
u(x, t)=\alpha x_{1}^{+}+o\left(|x|+|t|^{1 / 2}\right) \quad \text { in } B \cap\{t \leq 0\} .
$$


On the other hand, by Lemma A.2 in [13], there exists $\gamma \geq 0$ such that

$$
u^{-}(x, t)=\gamma x_{1}^{-}+o\left(|x|+|t|^{1 / 2}\right) \quad \text { in } \quad\{t \leq 0\} .
$$

Step A Assume $\gamma>0$. Then, there holds that

$$
u(x, t)=\alpha x_{1}^{+}-\gamma x_{1}^{-}+o\left(|x|+|t|^{1 / 2}\right) \quad \text { in } \quad\{t \leq 0\}
$$

and if $u_{0}=\lim _{n \rightarrow \infty} u_{\lambda_{n}}$ with $\lambda_{n} \rightarrow 0$ there holds that $u_{0}=\alpha x_{1}^{+}-\gamma x_{1}^{-}$in $\{t \leq 0\}$.

Now, we know that $u_{0}=\lim _{n \rightarrow \infty} u_{\lambda_{n}}$ satisfies that $u_{0}=\lim _{n \rightarrow \infty} u^{\delta_{n}}$ with $\delta_{n}:=\varepsilon_{j_{n}} / \lambda_{n} \rightarrow 0$ for a certain choice of $j_{n} \rightarrow \infty$. Moreover, $u^{\delta_{n}}=\left(u^{\varepsilon_{j_{n}}}\right)_{\lambda_{n}}$ and they are uniformly bounded in $\operatorname{Lip}(1,1 / 2)$ and solutions to $P_{\delta_{n}}\left(f_{\delta_{n}}\right)$ with $f_{\delta_{n}} \rightarrow 0$. (This argument was used frequently in previous articles. See for instance [9], [13]).

Thus, since $\gamma>0$, there holds that $\alpha^{2}-\gamma^{2}=2 M$. (This follows as in the proof of Proposition 5.1 in [9] with the necessary changes due to the presence of the forcing term $f_{\delta_{n}}$, with arguments as those used in [13] in the one phase case and in [14] in the two phase stationary case).

Step B Assume now $\gamma=0$. Then, there holds that $u_{0} \geq 0, \Delta u_{0}=u_{0 t}$ in $\left\{u_{0}>0\right\}, u_{0}$ is $\overline{\operatorname{Lip}(1,1} / 2)$ and $u_{0}=0$ on $\left\{x_{1}=0\right\}$. Thus, (see Corollary A.1 in [9])

$$
u_{0}(x, t)=\bar{\alpha} x_{1}^{-}+o\left(|x|+|t|^{1 / 2}\right) \quad \text { in } \quad\left\{x_{1}<0\right\} \cap\{t \leq 0\}
$$

with $\bar{\alpha} \geq 0$.

Let us see that we actually have

$$
u_{0}(x, t)=\bar{\alpha} x_{1}^{-} \quad \text { in } \quad\left\{x_{1}<0\right\} \cap\{t<0\} .
$$

In fact, as a consequence of Corollary 2.1 we know that $u_{0}$ is homogeneous. This is, $u_{0}(x, t)=$ $\frac{1}{\lambda} u_{0}\left(\lambda x, \lambda^{2} t\right)$, for $\lambda>0$. Then, for $x_{1}<0, t<0$,

$$
u_{0}(x, t)=\frac{1}{\lambda} u_{0}\left(\lambda x, \lambda^{2} t\right) \rightarrow \bar{\alpha} x_{1}^{-} \quad \text { as } \quad \lambda \rightarrow 0 .
$$

So that, (3.2) holds.

Thus, $u_{0}(x, t)=\alpha x_{1}^{+}+\bar{\alpha} x_{1}^{-}$, for $t \leq 0$, with $\alpha \geq 0$ independent of the sequence $\lambda_{n}$ and $\bar{\alpha} \geq 0$.

B.1 Assume that $\bar{\alpha}>0$. Let us see that $\bar{\alpha}=\alpha$ and that

$$
u(x, t)=\alpha x_{1}^{+}+\alpha x_{1}^{-}+o\left(|x|+|t|^{1 / 2}\right) \text { for } \quad\{t \leq 0\} .
$$

Let us first see that we may choose the sequence $\varepsilon_{j_{n}}$ in such a way that we also have

$$
B_{\delta_{n}}\left(u^{\delta_{n}}\right) \rightarrow \chi_{0} \quad * \text {-weakly in } L_{l o c}^{\infty}\left(\mathbb{R}^{N+1}\right)
$$

where $\chi_{0}=\lim \chi^{\lambda_{n}} *$-weakly in $L_{l o c}^{\infty}\left(\mathbb{R}^{N+1}\right)$ and $\chi=\lim B_{\varepsilon_{j}}\left(u^{\varepsilon_{j}}\right) *$-weakly in $L^{\infty}(\mathcal{D})$. Here, as above, $\delta_{n}=\frac{\varepsilon_{j_{n}}}{\lambda_{n}}$ and $u^{\delta_{n}}=\left(u^{\varepsilon_{j_{n}}}\right)_{\lambda_{n}}$.

In fact, for each $n \in \mathbb{N}$, let $\varepsilon_{j_{n}}$ be such that

$$
\left\|B_{\varepsilon_{j_{n}} / \lambda_{n}}\left(\left(u^{\varepsilon_{j_{n}}}\right)_{\lambda_{n}}\right)-\chi^{\lambda_{n}}\right\|_{L^{1}\left(Q_{r_{0} / \lambda_{n}}\right)}=\frac{1}{\lambda_{n}^{N+2}} \int_{Q_{r_{0}}}\left|B_{\varepsilon_{j_{n}}}\left(u^{\varepsilon_{j_{n}}}\right)-\chi\right| d x d t<\frac{1}{n}
$$

(we denote $Q_{r}=\left\{(x, t) /|x|<r,|t|<r^{2}\right\}$ ). This can be done since the subsequence may be chosen in such a way that $B_{\varepsilon_{j_{n}}}\left(u^{\varepsilon_{j_{n}}}\right) \rightarrow \chi$ strongly in $L^{1}\left(Q_{r_{0}}\right)$ for $r_{0}>0$ small. See [18], Proposition 4.1, where this result was proved in the one phase homogeneous case. For the two phase solutions of $P_{\varepsilon_{j}}\left(f_{\varepsilon_{j}}\right)$ the result follows similarly under the present assumptions. We omit the proof here. 
Let $\varphi \in L^{1} \cap L^{\infty}$ with compact support. Then, if $n$ is large enough so that supp $\varphi \subset Q_{r_{0} / \lambda_{n}}$,

$$
\begin{aligned}
& \left|\int\left(B_{\varepsilon_{j_{n}} / \lambda_{n}}\left(\left(u^{\varepsilon_{j_{n}}}\right)_{\lambda_{n}}\right)-\chi_{0}\right) \varphi d x d t\right| \leq \\
& \leq \int\left|B_{\varepsilon_{j_{n}} / \lambda_{n}}\left(\left(u^{\varepsilon_{j_{n}}}\right)_{\lambda_{n}}\right)-\chi^{\lambda_{n}}\right||\varphi| d x d t+\left|\int\left(\chi^{\lambda_{n}}-\chi_{0}\right) \varphi d x d t\right| \leq \frac{1}{n}\|\varphi\|_{\infty}+\eta
\end{aligned}
$$

for any $\eta>0$ if $n$ is large enough.

Now, if $\varphi \in L^{1}$ with compact support, we also get

$$
\int\left(B_{\varepsilon_{j_{n}} / \lambda_{n}}\left(\left(u^{\varepsilon_{j_{n}}}\right)_{\lambda_{n}}\right)-\chi_{0}\right) \varphi d x d t \rightarrow 0
$$

since $0 \leq B_{\delta_{n}}, \chi_{0} \leq M$.

Since, $\bar{\alpha}>0$ there holds that $u_{0}>0$ in $\left\{x_{1}<0\right\} \cap\{t \leq 0\}$. Thus, since $u_{0}=\lim u^{\delta_{n}}$ and $\chi_{0}=\lim B_{\delta_{n}}\left(u^{\delta_{n}}\right)$, necessarily $\chi_{0}=M$ in $\left\{x_{1}<0\right\} \cap\{t \leq 0\}$.

On the other hand, since $u>0$ in $B \cap\{t \leq 0\}$ there holds that $\chi=M$ in $B \cap\{t \leq 0\}$. Thus, $\chi_{0}=\lim \chi^{\lambda_{n}}=M$ in $\left\{x_{1}>0\right\} \cap\{t \leq 0\}$.

Thus, $B_{\delta_{n}}\left(u^{\delta_{n}}\right) \rightarrow M$ in $\{t \leq 0\}$. As in the proof of Proposition 5.3 in [9], using that the forcing term $f_{\delta_{n}}$ in the equation $P_{\delta_{n}}\left(f_{\delta_{n}}\right)$ satisfied by $u^{\delta_{n}}=\left(u^{\varepsilon_{j_{n}}}\right)_{\lambda_{n}}$ converges to 0 , we deduce that $\bar{\alpha}=\alpha$.

Now, let $\tilde{u}_{0}=\lim u_{\tilde{\lambda}_{n}}$ with $\tilde{\lambda}_{n} \rightarrow 0$. The argument above shows that $\tilde{u}_{0}=\alpha x_{1}^{+}+\tilde{\alpha} x_{1}^{-}$in $\{t \leq 0\}$ with $\tilde{\alpha} \geq 0$. Let us see that necessarily $\tilde{\alpha}>0$ and thus $\tilde{\alpha}=\alpha$.

In fact, by Corollary 2.1, we know that there exists $\delta$ independent of the sequences $\lambda_{n}$ and $\tilde{\lambda}_{n}$ such that

$$
\delta=\mathcal{W}\left(r, u_{0}, 1, \chi_{0}\right)=\mathcal{W}\left(r, \tilde{u}_{0}, 1, \tilde{\chi}_{0}\right) \quad \text { for every } \quad r>0 .
$$

Now, Corollary 2.2 states that

$$
\delta=\frac{1}{r^{2}} \int_{-4 r^{2}}^{-r^{2}} \int_{\mathbb{R}^{N}} 2 \chi_{0} G(x,-t) d x d t=\frac{1}{r^{2}} \int_{-4 r^{2}}^{-r^{2}} \int_{\mathbb{R}^{N}} 2 \tilde{\chi}_{0} G(x,-t) d x d t .
$$

Since, for $\{t \leq 0\}, \chi_{0} \equiv M$ and $0 \leq \tilde{\chi}_{0} \leq M$, necessarily $\tilde{\chi}_{0} \equiv M$ for $\{t \leq 0\}$.

As above, this implies that $\tilde{\alpha}=\alpha$.

Thus, if $\bar{\alpha}>0$,

$$
u(x, t)=\alpha x_{1}^{+}+\alpha x_{1}^{-}+o\left(|x|+|t|^{1 / 2}\right) \quad \text { for } \quad\{t \leq 0\} .
$$

Observe that in this case since we showed that $\alpha=\bar{\alpha}$ there holds that $\alpha>0$.

B.2 Assume $\bar{\alpha}=0$. This is, $u_{0}(x, t)=\alpha x_{1}^{+}$for $\{t \leq 0\}$. Let us see that

$$
u(x, t)=\alpha x_{1}^{+}+o\left(|x|+|t|^{1 / 2}\right) \quad \text { for } \quad\{t \leq 0\} .
$$

In fact, let $\tilde{u}_{0}=\lim u_{\tilde{\lambda}_{n}}$ with $\tilde{\lambda}_{n} \rightarrow 0$. Then, $\tilde{u}_{0}=\alpha x_{1}^{+}+\tilde{\alpha} x_{1}^{-}$in $\{t \leq 0\}$ with $\tilde{\alpha} \geq 0$.

If $\tilde{\alpha}>0$, the argument in B.1 with $\bar{\alpha}$ replaced by $\tilde{\alpha}$ shows that (3.4) holds with $\alpha>0$. In particular, $u_{0}(x, t)=\alpha x_{1}^{+}+\alpha x_{1}^{-}$for $\{t \leq 0\}$ with $\alpha>0$ which is a contradiction.

Thus, every blow up limit is $\alpha x_{1}^{+}$, for $\{t \leq 0\}$ and (3.5) holds.

This ends Step B.

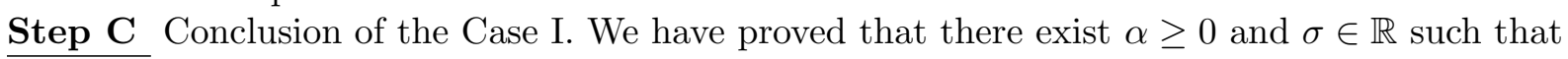

$$
u(x, t)=\alpha x_{1}^{+}+\sigma x_{1}^{-}+o\left(|x|+|t|^{1 / 2}\right) \quad \text { in } \quad\{t \leq 0\} .
$$

Let us characterize the values of $\alpha$ and $\sigma$. 
In Case B. 1 every blow up limit is $\alpha x_{1}^{+}+\alpha x_{1}^{-}$, for $\{t \leq 0\}$. As in [13], Proposition 5.2 we deduce that $0<\alpha \leq \sqrt{2 M}$.

In Case B.2 every blow up limit is $\alpha x_{1}^{+}$, for $\{t \leq 0\}$. As in [13], Proposition 5.1 we deduce that $\alpha=0$ or $\alpha=\sqrt{2 M}$.

Thus, if $\alpha>\sqrt{2 M}$, necessarily $\sigma<0$. And (1) in the statement holds.

If $0 \leq \alpha \leq \sqrt{2 M}$ there holds that $\sigma \geq 0$ since if not, $\sigma<0$ with $\alpha^{2}-\sigma^{2}=2 M$, so that $\alpha>\sqrt{2 M}$.

If $\alpha=\sqrt{2 M}$ both cases B.1 and B.2 are possible. Thus, $\sigma=0$ or $\sigma=\alpha=\sqrt{2 M}$. This proves (2) in the statement.

If $0<\alpha<\sqrt{2 M}$ the only possible case is B.1. Thus, $\sigma=\alpha$.

If $\alpha=0$, the only possible case is B.2. This is, $\sigma=\alpha=0$. This ends the proof of (3) in the statement.

CASE II: Assume now $B \cap\{t \leq 0\} \subset\{u \leq 0\}$ and $e_{1}$ is the exterior unit spatial normal to $B$ at $(0,0)$.

Since $u^{+}=0$ in $B \cap\{t \leq 0\}$ we get by applying Lemma A.2 in [13] that there exists $\alpha \geq 0$ such that

$$
u^{+}(x, t)=\alpha x_{1}^{+}+o\left(|x|+|t|^{1 / 2}\right) \quad \text { in } \quad\{t \leq 0\} .
$$

If $\alpha>0$ we get by applying the same ideas as in [14], Theorem 7.2, that the asymptotic development (3.1) holds with $\sigma \leq 0$ and $\alpha^{2}-\sigma^{2}=2 M$.

If, on the other hand, $\alpha=0$ we let $\lambda_{n} \rightarrow 0$ and $u_{0}=\lim u_{\lambda_{n}}$. Then, $u_{0} \leq 0$ in $\mathbb{R}^{N} \times(-\infty, 0]$, $\Delta u_{0}-\partial_{t} u_{0} \geq 0$ and $u_{0}(0,0)=0$. Therefore, $u_{0} \equiv 0$ in $\{t \leq 0\}$.

Then, the blow up limit $u_{0}$ is independent of the sequence $\lambda_{n}$, for $\{t \leq 0\}$. Therefore, the asymptotic development (3.1) holds with $\alpha=\sigma=0$.

\section{Applichtion II: Regularity of the fRee Boundary}

In this section we present further applications of the results in Section 2. They deal with the regularity of the boundary of $\{u>0\}$ in the stationary case including, in particular, regularity results for energy minimizers, and also with regularity results for travelling waves of a combustion model.

First, we consider a family $u^{\varepsilon_{j}}$ of stationary solutions to $P_{\varepsilon_{j}}\left(f_{\varepsilon_{j}}\right)$ such that $u^{\varepsilon_{j}}$ and $f_{\varepsilon_{j}}$ are uniformly bounded in $L^{\infty}$ norm. In [14] we prove that $u^{\varepsilon_{j}}$ are uniformly bounded in Lip norm. So that, the results of the present paper apply to this family. Let $u=\lim u^{\varepsilon_{j}}$ uniformly on compact subsets as $\varepsilon_{j} \rightarrow 0$. In [14] we prove that $u$ is a solution to

$$
\Delta u=f \chi_{\{u \neq 0\}} \text { in }\{u>0\} \cup\{u \leq 0\}^{\circ}
$$

where $f=\lim f_{\varepsilon_{j}} *$-weakly in $L^{\infty}$.

Moreover, in [14] we prove that, under suitable assumptions, $\partial\{u>0\}$ is smooth and $u$ is a classical solution to the following free boundary problem

$$
\begin{array}{ll}
\Delta u=f \chi_{\{u \neq 0\}} & \text { in }\{u>0\} \cup\{u \leq 0\}^{\circ}, \\
\left|\nabla u^{+}\right|^{2}-\left|\nabla u^{-}\right|^{2}=2 M & \text { on } \partial\{u>0\} .
\end{array}
$$

The purpose of this section is to state some theorems on the regularity of the free boundary $\partial\{u>0\}$ that are proved in [14] for which the results in this paper are an essential tool. 
In fact, assume $u$ is defined in $B_{R}\left(x_{0}\right)$ with $x_{0} \in \partial\{u>0\}$. Let $\chi=\lim B_{\varepsilon_{j}}\left(u^{\varepsilon_{j}}\right) *$-weakly in $L^{\infty}\left(B_{R}\left(x_{0}\right)\right)$ and

$$
\delta\left(x_{0}\right)=\lim _{r \rightarrow 0^{+}} \frac{1}{r^{2}} \int_{-4 r^{2}}^{-r^{2}} \int_{\mathbb{R}^{N}}\left(|\nabla(u \psi)|^{2}+2 \psi^{2} \chi+\frac{1}{2} \frac{(u \psi)^{2}}{t}\right) G\left(x-x_{0},-t\right) d x d t,
$$

where $G(x, t)=\frac{1}{(4 \pi t)^{N / 2}} \exp \left(-\frac{|x|^{2}}{4 t}\right)$ and $\psi$ is any function satisfying that $\psi \in C_{0}^{\infty}\left(B_{R}\left(x_{0}\right)\right)$, $0 \leq \psi \leq 1, \psi \equiv 1$ in $B_{R / 2}\left(x_{0}\right)$.

In the next three theorems, we assume that, in $B_{R}\left(x_{0}\right), u^{+}$is uniformly nondegenerate. This property holds in many applications (see, for instance, Theorems 4.4 and 4.5). By uniform nondegeneracy we mean that there exists $c>0$ such that

$$
u^{+}(x) \geq c \operatorname{dist}(x,\{u \leq 0\}) .
$$

As mentioned before, we have the following result,

Theorem 4.1 (Theorem 9.7 in [14]). There holds that $\delta\left(x_{0}\right)=3 M$ if and only if the free boundary is $C^{1, \alpha}$ in a neighborhood of $x_{0}$. This implies that $u$ is a classical solution to the free boundary problem $E(f)$ in a neighborhood of $x_{0}$.

In the proof of the next two theorems we use the result on asymptotic developments (Theorem 3.1). In case $N=2$ we use that blow up limits are homogeneous as proved in Corollary 2.1

Theorem 4.2 (Theorem 9.5 in [14]). If $x_{0}$ is a regular point from the right and $\delta\left(x_{0}\right)<6 M$ then, the free boundary is a $C^{1, \alpha}$ surface in a neighborhood of $x_{0}$. Moreover, $u$ is a classical solution to the free boundary problem $E(f)$ in a neighborhood of $x_{0}$.

If $N=2$ the same result holds without assuming that $x_{0}$ is a regular point from the right.

Theorem 4.3 (Theorem 9.6 in [14]). If $x_{0}$ is a regular point from the right and, in addition, $\limsup _{r \rightarrow 0} \frac{\left|B_{r}\left(x_{0}\right) \cap\{u \leq 0\}\right|}{\left|B_{r}\left(x_{0}\right)\right|}>0$ then, the free boundary is a $C^{1, \alpha}$ surface in a neighborhood of $x_{0}$. Moreover, $u$ is a classical solution to the free boundary problem $E(f)$ in a neighborhood of $x_{0}$.

If $N=2$ the same result holds without assuming that $x_{0}$ is a regular point from the right.

We say that a free boundary point $x_{0}$ is regular from the right if there is a ball contained in $\{u>0\}$ that is tangent to the free boundary at $x_{0}$. Note that regular points from the right are dense in the free boundary.

We recall that the examples in [13] show that the free boundary condition may not hold at any free boundary point. In fact, $u^{+}$may degenerate or the density of $\{u \leq 0\}$ may be zero at a boundary point. These situations may appear even at points that are regular from the right. Thus, some extra assumption is needed if one wants to show that $u$ is a solution to $E(f)$.

The results in the present paper are also used in [14] to obtain the following regularity results for energy minimizers and for travelling waves of a combustion model.

The first of these results is

Theorem 4.4 (Theorem 10.2 in [14]). Let $\Omega \subset \mathbb{R}^{N}$ be a bounded domain and let $\phi_{\varepsilon} \in H^{1}(\Omega)$ be such that $\left\|\phi_{\varepsilon}\right\|_{H^{1}(\Omega)} \leq \mathcal{A}_{1}$. Let $f^{\varepsilon} \in L^{\infty}(\Omega)$ such that $\left\|f^{\varepsilon}\right\|_{L^{\infty}(\Omega)} \leq \mathcal{A}_{2}$. Let $u^{\varepsilon} \in H^{1}(\Omega)$ be a minimizer of the energy

$$
J_{\varepsilon}(v)=\int_{\Omega} \frac{1}{2}|\nabla v|^{2}+B_{\varepsilon}(v)+f^{\varepsilon} v
$$

among functions $v \in H^{1}(\Omega)$ such that $v=\phi_{\varepsilon}$ on $\partial \Omega$. Here $B_{\varepsilon}(s)=\int_{0}^{s} \beta_{\varepsilon}(\tau) d \tau$. 
Then, the functions $u^{\varepsilon}$ are stationary solutions to $P_{\varepsilon}\left(f_{\varepsilon}\right)$ in $\Omega$ and they are uniformly bounded in $\operatorname{Lip}\left(\Omega^{\prime}\right)$ for every $\Omega^{\prime} \subset \subset \Omega$.

Let $u=\lim u^{\varepsilon_{j}}$ and $f=\lim f_{\varepsilon_{j}}$ with $\varepsilon_{j} \rightarrow 0$. Then, there is a subset $\mathcal{R}$ of the free boundary $\Omega \cap \partial\{u>0\}$ which is locally a $C^{1, \alpha}$ surface and $u$ is a classical solution to the free boundary problem $E(f)$ in a neighborhood of $\mathcal{R}$. Moreover, $\mathcal{R}$ is open and dense in $\Omega \cap \partial\{u>0\}$ and the remainder of the free boundary has $(N-1)$-dimensional Hausdorff measure zero.

In dimensions 2 and 3 we have $\mathcal{R}=\Omega \cap \partial\{u>0\}$.

In addition, in any dimension, if $u \geq 0$ and $f \in C_{\mathrm{loc}}^{k, \alpha}$ (resp. analytic) then, $\mathcal{R} \in C_{\mathrm{loc}}^{k+2, \alpha}$ (resp. analytic).

The last application we present is the following

Theorem 4.5 (Theorem 10.1 in [14]). Let $x=\left(x_{1}, y\right) \in \Omega=\mathbb{R} \times \Sigma$, with $\Sigma \subset \mathbb{R}^{N-1}$ a smooth bounded domain, let a be a continuous positive function on $\bar{\Sigma}$ and let $0<\sigma<1$ be given.

Consider travelling wave solutions to the following combustion model

$$
\Delta v^{\varepsilon}-a(y) v_{t}^{\varepsilon}=\beta_{\varepsilon}\left(v^{\varepsilon}\right),
$$

where $\beta_{\varepsilon}$ is as before with $\beta^{\prime}(0)>0$. This is, $v^{\varepsilon}(x, t)=u^{\varepsilon}\left(x_{1}+c^{\varepsilon} t, y\right)$, with $u^{\varepsilon}$ solutions to

$$
\begin{gathered}
\Delta u^{\varepsilon}-c^{\varepsilon} a(y) u_{x_{1}}^{\varepsilon}=\beta_{\varepsilon}\left(u^{\varepsilon}\right) \quad \text { in } \Omega, \\
u^{\varepsilon}(-\infty, y)=(1-\sigma)^{-1}, \quad u^{\varepsilon}(+\infty, y)=0 \quad \text { in } \Sigma, \\
\frac{\partial u^{\varepsilon}}{\partial \eta}=0 \quad \text { on } \mathbb{R} \times \partial \Sigma,
\end{gathered}
$$

for some suitable $c^{\varepsilon}$.

Let $u=\lim u^{\varepsilon_{j}}\left(\varepsilon_{j} \rightarrow 0\right)$. Then, there is a subset $\mathcal{R}$ of the free boundary $\Omega \cap \partial\{u>0\}$ which is locally a $C^{1, \alpha}$ surface and $u$ is a classical solution to the free boundary problem $E(f)$ in a neighborhood of $\mathcal{R}\left(f=c a(y) u_{x_{1}}\right.$ with $\left.c=\lim c^{\varepsilon_{j}}\right)$. Moreover, $\mathcal{R}$ is open and dense in $\Omega \cap \partial\{u>0\}$ and the remainder of the free boundary has $(N-1)$-dimensional Hausdorff measure zero.

In dimension 2 we have $\mathcal{R}=\Omega \cap \partial\{u>0\}$.

In addition, in any dimension, if $a \in C_{\mathrm{loc}}^{k, \alpha}$ (resp. analytic) then, $\mathcal{R} \in C_{\mathrm{loc}}^{k+2, \alpha}$ (resp. analytic).

We remark that this problem was first studied in [2], where the authors obtained existence of $\left(u^{\varepsilon}, c_{\varepsilon}\right)$, strict monotonicity in the $x_{1}$ direction, uniform Lipschitz estimates and uniform nondegeneracy of the family $u^{\varepsilon}$, as well as uniform estimates of the velocities $c_{\varepsilon}$.

The proof of Theorem 4.5 relies on the fact that the density of the zero set is positive at every free boundary point. We obtain this density property by a contradiction argument by means of a delicate discussion on the consequences of the equality (2.19) in Corollary 2.2. In dimension 2 we also use that blow up limits are homogeneous as proved in Corollary 2.1.

\section{REFERENCES}

[1] Athanasopoulos, I.; Caffarelli, L. A.; Salsa, S. Phase transition problems of parabolic type: flat free boundaries are smooth, Comm. Pure Appl. Math. 51 (1), (1998), 77-112.

[2] H. Berestycki, L.A. Caffarelli, L. Nirenberg, Uniform estimates for regularization of free boundary problems, "Analysis and Partial Differential Equations" (Cora Sadosky, ed.), Lecture Notes in Pure and Applied Mathematics, vol. 122, Marcel Dekker, New York, 1990, 567-619.

[3] J. D. Buckmaster, G. S. S. Ludford, Theory of Laminar Flames, Cambridge University Press, Cambridge, 1982. 
[4] L.A. Caffarelli, A Harnack inequality approach to the regularity of free boundaries. Part I: Lipschitz free boundaries are $C^{1, \alpha}$, Rev. Matem. Iberoamericana 3 (2), (1987), 139-162.

[5] L. A. Caffarelli, A Harnack inequality approach to the regularity of free boundaries. Part II: Flat free boundaries are Lipschitz, Comm. Pure and Appl. Math. 42, (1989), 55-78.

[6] L. A. Caffarelli, Uniform Lipschitz Regularity of a Singular Perturbation Problem, Diff. Int. Eqs. 8 (7), (1995), 1585-1590.

[7] L.A. Caffarelli, D. Jerison, C.E. Kenig, Global energy minimizers for free boundary problems and full regularity in three dimensions, Noncompact problems at the intersection of geometry, analysis, and topology, Contemp. Math. 350, 83-97, Amer. Math. Soc., Providence, RI, 2004.

[8] L.A. Caffarelli and C. Kenig, Gradient estimates for variable coefficient parabolic equations and singular perturbation problems, Amer. J. Math. 120 (2), (1998), 391-439.

[9] L. A. Caffarelli, C. Lederman, N. Wolanski, Uniform estimates and limits for a two phase parabolic singular perturbation problem, Indiana Univ. Math. J. 46 (2), (1997), 453-490.

[10] L. A. Caffarelli, A. Petrosyan, H. Shahgholian, Regularity of a free boundary in parabolic potential theory, J. Amer. Math. Soc. 17 (4), (2004), 827-869

[11] L.A. Caffarelli, J.L. Vazquez, A free boundary problem for the heat equation arising in flame propagation, Trans. Amer. Math. Soc. 347, (1995), 411-441.

[12] P. Fife. Some nonclassical trends in parabolic and parabolic-like evolutions. Trends in nonlinear analysis, 153-191, Springer, Berlin, 2003.

[13] C. Lederman, N. Wolanski, Singular perturbation in a nonlocal diffusion problem, Communications in PDE 31(2), (2006), 195-241.

[14] C. Lederman, N. Wolanski, A two phase elliptic singular perturbation problem with a forcing term, preprint.

[15] J. L. Vázquez, The free boundary problem for the heat equation with fixed gradient condition, Proceedings International Conference on free boundary problems and applications, Zakopane, Poland, 1995.

[16] G. S. Weiss, Partial regularity for weak solutions of an elliptic free boundary problem, Comm. Partial Differential Equations 23 (3\&4) (1998), 439-455.

[17] G. S. Weiss, A homogeneity property improvement approach to the obstacle problem, Invent. Math. 138, (1999), 23-50.

[18] G. S. Weiss, A singular limit arising in combustion theory: fine properties of the free boundary, Calculus of Variations and Partial Diff. Equations 17 (3), (2003), 311-340.

Departamento de Matemática, Facultad de Ciencias Exactas y Naturales, Universidad de Buenos Aires, (1428) Buenos Aires, Argentina.

E-mail address, Claudia Lederman: clederma@dm.uba.ar

E-mail address, Noemi Wolanski: wolanski@dm.uba.ar 